\title{
Best constants in Sobolev and Gagliardo-Nirenberg inequalities on graded groups and ground states for higher order nonlinear subelliptic equations
}

\author{
Michael Ruzhansky ${ }^{1,2} \cdot$ Niyaz Tokmagambetov $^{1,3} \cdot$ Nurgissa Yessirkegenov $^{1,4,5}$
}

Received: 7 November 2019 / Accepted: 31 July 2020 / Published online: 16 September 2020

(c) The Author(s) 2020

\begin{abstract}
In this paper the dependence of the best constants in Sobolev and Gagliardo-Nirenberg inequalities on the precise form of the Sobolev space norm is investigated. The analysis is carried out on general graded Lie groups, thus including the cases of $\mathbb{R}^{n}$, Heisenberg, and general stratified Lie groups, in all these cases the results being new. The Sobolev norms may be defined in terms of Rockland operators, i.e. the hypoelliptic homogeneous left-invariant differential operators on the group. The best constants are expressed in the variational form as well as in terms of the ground state solutions of the corresponding nonlinear subelliptic equations. The orders of these equations can be high depending on the Sobolev space order in the Sobolev or Gagliardo-Nirenberg inequalities, or may be fractional. Applications are obtained also to equations with lower order terms given by different hypoelliptic operators. Already in the case of $\mathbb{R}^{n}$, the obtained results extend the classical relations by Weinstein (Commun Math Phys 87(4):567-576 (1982/1983)) to a wide range of nonlinear elliptic equations of high orders with elliptic low order terms and a wide range of interpolation inequalities of Gagliardo-Nirenberg type. However, the proofs are different from those in Weinstein (Commun Math Phys 87(4):567-576 (1982/1983)) because of the impossibility of using the rearrangement inequalities already in the setting of the Heisenberg group. The considered class of graded groups is the most general class of nilpotent Lie groups where one can still consider hypoelliptic homogeneous invariant differential operators and the corresponding subelliptic differential equations.
\end{abstract}

Mathematics Subject Classification $35 \mathrm{~J} 35 \cdot 35 \mathrm{G} 20 \cdot 22 \mathrm{E} 30 \cdot 43 \mathrm{~A} 80$

Communicated by N. Trudinger.

The authors were supported by the FWO Odysseus 1 Grant G.0H94.18N: Analysis and Partial Differential Equations. MR was supported by the EPSRC Grant EP/R003025/1 and by the Leverhulme Research Grant RPG-2017-151. NT was supported by the MESRK Grant AP08053051. NY was supported by the MESRK Grant AP05133271. No new data was collected or generated during the course of research.

$\bowtie$ Michael Ruzhansky

Michael.Ruzhansky@UGent.be

Extended author information available on the last page of the article 


\section{Contents}

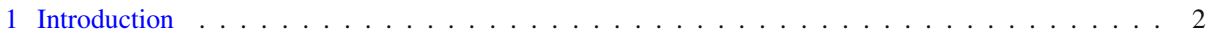

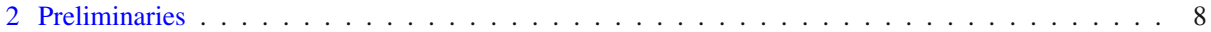

3 Gagliardo-Nirenberg inequality on graded Lie groups _. . . . . . . . . . . . . . . . . 9

4 Existence of least energy solutions for a class of nonlinear subelliptic equations . . . . . . . . . . . 11

5 Best constants in the Gagliardo-Nirenberg inequalities . . . . . . . . . . . . . . . . . . . . . . 17

6 Best constants in the Sobolev inequalities . . . . . . . . . . . . . . . . . . . . . . 20

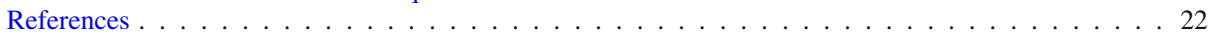

\section{Introduction}

The Gagliardo-Nirenberg inequality goes back to works of Gagliardo [12] and Nirenberg [22] where it was shown that the inequality

$$
\int_{\mathbb{R}^{n}}|u|^{q} d x \leq C\left(\int_{\mathbb{R}^{n}}|\nabla u|^{2} d x\right)^{\frac{n(q-2)}{4}}\left(\int_{\mathbb{R}^{n}}|u|^{2} d x\right)^{\frac{2 q-n(q-2)}{4}}
$$

holds for all $u \in H^{1}\left(\mathbb{R}^{n}\right)$. Here one can take

$$
\left\{\begin{aligned}
2 & \leq q<\infty \text { for } n=2 \\
2 \leq q & \leq \frac{2 n}{n-2} \text { for } n \geq 3
\end{aligned}\right.
$$

Weinstein [37] obtained an expression for the best constant in the inequality (1.1), relating it to the ground states (least energy solutions) of the nonlinear Schrödinger equation

$$
-\Delta u+u=|u|^{q-2} u, \quad u \in H^{1}\left(\mathbb{R}^{n}\right) .
$$

This result has numerous applications (there are 430 citations to [37] on MathSciNet), for example to further properties of the critical nonlinear Schrödinger equation (1.2), [19,20], and to many other problems.

On the Heisenberg group $\mathbb{H}^{N}$, the subelliptic Gagliardo-Nirenberg inequality takes the form

$$
\int_{\mathbb{H}^{N}}|u|^{q} d x \leq C\left(\int_{\mathbb{H}^{N}}\left|\nabla_{H} u\right|^{2} d x\right)^{\frac{Q(q-2)}{4}}\left(\int_{\mathbb{H}^{N}}|u|^{2} d x\right)^{\frac{2 q-Q(q-2)}{4}},
$$

where $\nabla_{H}$ is a horizontal gradient, $Q=2 N+2$ is the homogeneous dimension of $\mathbb{H}^{N}$, $2<q<2+\frac{2}{N}$. In [5], the best constant for the Gagliardo-Nirenberg inequality (1.3) on the Heisenberg group was expressed in terms of the ground state solutions of the subelliptic equation

$$
-\triangle_{H} u+u=|u|^{q-2} u, \quad u \in H^{1}\left(\mathbb{H}^{N}\right),
$$

where $\triangle_{H}$ is the sub-Laplacian on $\mathbb{H}^{N}$, and $H^{1}\left(\mathbb{H}^{N}\right)$ is the Sobolev space on $\mathbb{H}^{N}$ with the norm

$$
\|u\|:=\left(\int_{\mathbb{H}^{N}}\left(\left|\nabla_{H} u\right|^{2}+|u|^{2}\right) d x\right)^{1 / 2} .
$$

One of the aims of this paper is to answer the following questions:

- How do the best constants in the Gagliardo-Nirenberg inequalities (1.1), (1.3) depend on the precise formula for the Sobolev norms? For example, if we replace $\|u\|_{\dot{H}^{1}\left(\mathbb{R}^{n}\right)}=$ 
$\|\nabla u\|_{L^{2}\left(\mathbb{R}^{n}\right)}$ by the equivalent norm $\left\|(-\Delta)^{1 / 2} u\right\|_{L^{2}\left(\mathbb{R}^{n}\right)}$ or by the equivalent norms $\left\|\left((-1)^{m} \sum_{j=1}^{n} \partial_{x_{j}}^{2 m}\right)^{\frac{1}{2 m}} u\right\|_{L^{2}\left(\mathbb{R}^{n}\right)}$, and similarly for the Heisenberg group, how does it influence the best constants in (1.1), (1.3) and the nonlinear Eqs. (1.2), (1.4)?

- What can be said about more general Gagliardo-Nirenberg inequalities? For example, when the first order Sobolev norm in (1.1), (1.3) is replaced by higher order Sobolev norms? Also, when the $L^{2}$-norms on the right hand sides in (1.1), (1.3) are replaced by appropriate $L^{p}$-norm for other values of $p$ ?

From this perspective, our results will be new already in the classical Euclidean setting of $\mathbb{R}^{n}$.

A natural setting for our analysis will be that of graded Lie groups as developed by Folland and Stein [11]. This is the largest class of homogeneous nilpotent Lie groups admitting homogeneous hypoelliptic left-invariant differential operators ([18,36], see also a discussion in [10, Section 4.1]). These operators are called Rockland operator, after Helffer and Nourrigat's resolution [15] of the Rockland conjecture. Thus, our setting will include the higher order operators on $\mathbb{R}^{n}$ as well as higher order hypoelliptic invariant differential operators on the Heisenberg group, on general stratified groups, and on general graded Lie groups. In all these cases, thus including $\mathbb{R}^{n}$, Heisenberg, and stratified groups, the results of the paper appear to be new.

We also note that the Rockland operators on graded Lie groups appear naturally in the analysis of subelliptic operators on manifolds, starting with the seminal paper of Rothschild and Stein [24].

Thus, let $\mathbb{G}$ be a graded Lie group, i.e. a connected simply connected Lie group such that its Lie algebra $\mathfrak{g}$ admits a decomposition

$$
\mathfrak{g}=\bigoplus_{\ell=1}^{\infty} \mathfrak{g}_{\ell},
$$

where the $\mathfrak{g}_{\ell}, \ell=1,2, \ldots$, are vector subspaces of $\mathfrak{g}$, all but finitely many equal to $\{0\}$, and satisfying

$$
\left[\mathfrak{g}_{\ell}, \mathfrak{g}_{\ell^{\prime}}\right] \subset \mathfrak{g}_{\ell+\ell^{\prime}} \quad \forall \ell, \ell^{\prime} \in \mathbb{N} .
$$

Such groups are then necessarily nilpotent and homogeneous, they can be identified with $\mathbb{R}^{n}$ through the exponential mapping, with $n$ being the topological dimension of $\mathbb{G}$, with the Haar measure on $\mathbb{G}$ given by the Lebesgue measure on $\mathbb{R}^{n}$ through this identification. A family of dilations of a Lie algebra $\mathfrak{g}$ is a family of linear mappings of the form

$$
D_{r}=\operatorname{Exp}(A \ln r)=\sum_{k=0}^{\infty} \frac{1}{k !}(\ln (r) A)^{k},
$$

where $A$ is a diagonalisable linear operator on the Lie algebra $\mathfrak{g}$ with positive eigenvalues, and each $D_{r}$ is a morphism of $\mathfrak{g}$, that is, a linear mapping from $\mathfrak{g}$ to itself satisfying

$$
\forall X, Y \in \mathfrak{g}, r>0,\left[D_{r} X, D_{r} Y\right]=D_{r}[X, Y],
$$

where $[X, Y]:=X Y-Y X$ is the Lie bracket. If $v_{1}, \ldots, v_{n}$ are weights of the dilations, i.e. the eigenvalues of the matrix $A$, then the group's dilations are defined through the exponential mapping by

$$
D_{r}(x)=r x:=\left(r^{\nu_{1}} x_{1}, \ldots, r^{v_{n}} x_{n}\right), \quad x=\left(x_{1}, \ldots, x_{n}\right) \in \mathbb{G}, \quad r>0 .
$$


The homogeneous dimension of $\mathbb{G}$ is defined by

$$
Q:=\operatorname{Tr} A=v_{1}+\cdots+v_{n} .
$$

In Sect. 2 we will give a short overview of graded Lie groups, but we can mention here that we have $\mathbb{R}^{n}$ as a special case with all $v_{j}=1$, or the stratified groups when $\mathfrak{g}$ is generated by its first stratum $\mathfrak{g}_{1}$ through the iterative application of commutators.

Let $\mathcal{R}$ be a positive Rockland operator on $\mathbb{G}$, i.e. a homogeneous hypoelliptic left-invariant differential operator, positive in the operator sense. Such operators always exist. For example, for the Heisenberg group, the sub-Laplacian and its powers are Rockland operators. If $\mathbb{G}$ is a stratified Lie group with a basis $X_{1}, \ldots, X_{k} \in \mathfrak{g}_{1}$ of $\mathfrak{g}_{1}$, the operators

$$
\mathcal{R}=(-1)^{m} \sum_{j=1}^{k} a_{j} X_{j}^{2 m}, \quad a_{j}>0,
$$

are positive Rockland operators for any $m \in \mathbb{N}$, yielding the sub-Laplacian for $m=1$. More generally, for any graded Lie group $\mathbb{G} \sim \mathbb{R}^{n}$ with dilation weights $v_{1}, \ldots, v_{n}$ and a basis $X_{1}, \ldots, X_{n}$ of the Lie algebra $\mathfrak{g}$ of $\mathbb{G}$ satisfying

$$
D_{r} X_{j}=r^{v_{j}} X_{j}, \quad j=1, \ldots, n, r>0,
$$

the operator

$$
\mathcal{R}=\sum_{j=1}^{n}(-1)^{\frac{\nu_{0}}{v_{j}}} a_{j} X_{j}^{2 \frac{\nu_{0}}{v_{j}}}, \quad a_{j}>0,
$$

is a Rockland operator of homogeneous degree $2 v_{0}$, if $v_{0}$ is any common multiple of $v_{1}, \ldots, v_{n}$. There are other examples of Rockland operators that can be adapted to special selections of vector fields generating the Lie algebra in special ways, such as for example the vector fields from the first stratum on the stratified Lie groups. We refer to [10, Section 4.1.2] for other examples and a detailed discussion of Rockland operators.

Sobolev spaces associated to positive Rockland operators on graded Lie groups have been analysed in [9] and in [10, Section 4.4]. In particular, for a positive Rockland operator of homogeneous degree $v \in \mathbb{N}$, for $a>0$ and $1<p<\infty$, we can define the homogeneous and inhomogeneous Sobolev spaces, respectively, by the norms

$$
\|u\|_{\dot{L}_{a, \mathcal{R}}^{p}(\mathbb{G})}:=\left\|\mathcal{R}^{\frac{a}{v}} u\right\|_{L^{p}(\mathbb{G})} \text { and }\|u\|_{L_{a, \mathcal{R}}^{p}(\mathbb{G})}:=\left(\left\|\mathcal{R}^{\frac{a}{v}} u\right\|_{L^{p}(\mathbb{G})}^{p}+\|u\|_{L^{p}(\mathbb{G})}^{p}\right)^{\frac{1}{p}}
$$

If $\mathbb{G}=\mathbb{R}^{n}$ and $\mathcal{R}$ is a homogeneous elliptic operator with constant coefficients, these spaces coincide with the usual Sobolev spaces on $\mathbb{R}^{n}$. If $\mathbb{G}$ is a stratified Lie group and $\mathcal{R}$ is a positive sub-Laplacian, then these Sobolev spaces have been analysed by Folland in [8]. For an extensive analysis of these Sobolev spaces on general graded Lie group we refer to [9] or [10, Section 4.4]. In particular, it was shown that these spaces are independent of the choice of a positive operator $\mathcal{R}$, so that we can drop the subscript $\mathcal{R}$ in their notation, abbreviating them to $\dot{L}_{a}^{p}$ and $L_{a}^{p}$, respectively. While these spaces are independent of $\mathcal{R}$, the particular norms in (1.6) do depend on it.

The starting point of our analysis in this paper is the following Gagliardo-NirenbergSobolev inequality on graded groups:

- (Gagliardo-Nirenberg inequality) Let $\mathbb{G}$ be a graded Lie group of homogeneous dimension $Q$ and let $\mathcal{R}_{1}$ and $\mathcal{R}_{2}$ be positive Rockland operators of homogeneous degrees $v_{1}$ 
and $v_{2}$, respectively. Let $a_{1}>a_{2} \geq 0,1<p<\frac{Q}{a_{1}}$ and $\frac{p Q}{Q-a_{2} p} \leq q \leq \frac{p Q}{Q-a_{1} p}$. Then there exists a constant $C>0$ such that

$$
\int_{\mathbb{G}}|u(x)|^{q} d x \leq C\left(\int_{\mathbb{G}}\left|\mathcal{R}_{1}^{\frac{a_{1}}{\nu_{1}}} u(x)\right|^{p} d x\right)^{\frac{Q(q-p)-a_{2} p q}{\left(a_{1}-a_{2}\right) p^{2}}}\left(\int_{\mathbb{G}}\left|\mathcal{R}_{2}^{\frac{a_{2}}{\nu_{2}}} u(x)\right|^{p} d x\right)^{\frac{a_{1} p q-Q(q-p)}{\left(a_{1}-a_{2}\right) p^{2}}}
$$

holds for all $u \in \dot{L}_{a_{1}}^{p}(\mathbb{G}) \cap \dot{L}_{a_{2}}^{p}(\mathbb{G})$.

The freedom of working with two different Rockland operators $\mathcal{R}_{1}, \mathcal{R}_{2}$ in (1.7) leads to the possibility of considering two different (hypo)elliptic operators in (1.4). Throughout this paper we will often abbreviate the notation by writing

$$
L_{a_{1}, a_{2}}^{p}(\mathbb{G}):=\dot{L}_{a_{1}}^{p}(\mathbb{G}) \cap \dot{L}_{a_{2}}^{p}(\mathbb{G}) .
$$

The inequality (1.7) will be established in Sect. 3. Consequently, the question arises of what is the best constant $C$ in this inequality, which we may denote by $C_{G N, \mathcal{R}_{1}, \mathcal{R}_{2}}=$ $C_{G N, \mathcal{R}_{1}, \mathcal{R}_{2}, a_{1}, a_{2}, p, q}$ since it depends on the operators $\mathcal{R}_{1}, \mathcal{R}_{2}$ as well as on the indices $a_{1}, a_{2}, p, q$. The related question is of the best constant in the Sobolev (embedding) inequality

$$
\left(\int_{\mathbb{G}}|u(x)|^{q} d x\right)^{\frac{p}{q}} \leq C \int_{\mathbb{G}}\left(\left|\mathcal{R}^{\frac{a}{v}} u(x)\right|^{p}+|u(x)|^{p}\right) d x,
$$

where $u \in L_{a}^{p}(\mathbb{G})$, see [10, Theorem 4.4.28] for its proof in the setting of general graded Lie groups.

In this paper we will show that both the Sobolev inequality (1.8) and the GagliardoNirenberg inequality (1.7) are related to the following Schrödinger equation with the power nonlinearities:

$$
\mathcal{R}_{1}^{\frac{a_{1}}{\nu_{1}}}\left(\left|\mathcal{R}_{1}^{\frac{a_{1}}{\nu_{1}}} u\right|^{p-2} \mathcal{R}_{1}^{\frac{a_{1}}{\nu_{1}}} u\right)+\mathcal{R}_{2}^{\frac{a_{2}}{\nu_{2}}}\left(\left|\mathcal{R}_{2}^{\frac{a_{2}}{\nu_{2}}} u\right|^{p-2} \mathcal{R}_{2}^{\frac{a_{2}}{\nu_{2}}} u\right)=|u|^{q-2} u
$$

Moreover, these inequalities are related to the variational problem

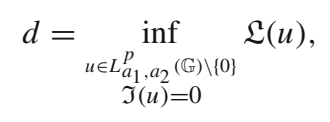

for functionals

$$
\mathfrak{L}(u)=\frac{1}{p} \int_{\mathbb{G}}\left|\mathcal{R}_{1}^{\frac{a_{1}}{\nu_{1}}} u(x)\right|^{p} d x+\frac{1}{p} \int_{\mathbb{G}}\left|\mathcal{R}_{2}^{\frac{a_{2}}{\nu_{2}}} u(x)\right|^{p} d x-\frac{1}{q} \int_{\mathbb{G}}|u(x)|^{q} d x
$$

and

$$
\Im(u):=\int_{\mathbb{G}}\left(\left|\mathcal{R}_{1}^{\frac{a_{1}}{v_{1}}} u(x)\right|^{p}+\left|\mathcal{R}_{2}^{\frac{a_{2}}{\nu_{2}}} u(x)\right|^{p}-|u(x)|^{q}\right) d x .
$$

Thus, in this paper we will show that

- (Existence of ground state solutions) Let $a_{1}>a_{2} \geq 0,1<p<\frac{Q}{a_{1}}$ and $\frac{p Q}{Q-a_{2} p}<q<$ $\frac{p Q}{Q-a_{1} p}$. Then the nonlinear Schrödinger type equation (1.9) has a least energy solution $\phi \in \dot{L}_{a_{1}}^{p}(\mathbb{G}) \cap \dot{L}_{a_{2}}^{p}(\mathbb{G})$, i.e. a solution $\phi$ such that $d=\mathfrak{L}(\phi)$.

- (Best constants in Sobolev embeddings) Let $a>0,1<p<\frac{Q}{a}, p<q<\frac{p Q}{Q-a p}$, let $\phi$ be a least energy solution of

$$
\mathcal{R}^{\frac{a}{v}}\left(\left|\mathcal{R}^{\frac{a}{v}} u\right|^{p-2} \mathcal{R}^{\frac{a}{v}} u\right)+|u|^{p-2} u=|u|^{q-2} u,
$$


and let $d=\mathfrak{L}(\phi)$. Let $C_{S, \mathcal{R}, a, p, q}$ be the best constant in the Sobolev embedding theorem, i.e. the smallest constant $C$ in the inequality (1.8). Then we have

$$
C_{S, \mathcal{R}, a, p, q}=\left(\frac{a p q}{a p q-Q(q-p)} \int_{\mathbb{G}}|\phi(x)|^{p} d x\right)^{\frac{p-q}{q}}=\left(\frac{p q}{q-p} d\right)^{\frac{p-q}{q}} .
$$

- (Best constants in Gagliardo-Nirenberg inequalities) Let $a_{1}>a_{2} \geq 0,1<p<\frac{Q}{a_{1}}$, $\frac{p Q}{Q-a_{2} p}<q<\frac{p Q}{Q-a_{1} p}$, let $\phi$ be a least energy solution of (1.9), and let $d=\mathfrak{L}(\phi)$. Let $C_{G N, \mathcal{R}_{1}, \mathcal{R}_{2}, a_{1}, a_{2}, p, q}$ be the best constant in the Gagliardo-Nirenberg inequality, i.e. the smallest constant $C$ in the inequality (1.7). Then we have

$$
\begin{aligned}
& C_{G N, \mathcal{R}_{1}, \mathcal{R}_{2}, a_{1}, a_{2}, p, q} \\
& =\frac{\left(a_{1}-a_{2}\right) p q}{a_{1} p q-Q(q-p)}\left(\frac{Q(q-p)-a_{2} p q}{a_{1} p q-Q(q-p)}\right)^{\frac{a_{2} p q-Q(q-p)}{\left(a_{1}-a_{2}\right) p^{2}}}\left(\frac{a_{1} p q-Q(q-p)}{\left(a_{1}-a_{2}\right)(q-p)} d\right)^{\frac{p-q}{p}} .
\end{aligned}
$$

The obtained results provide new insights already in the case of $\mathbb{G}=\mathbb{R}^{n}$ in view of the arbitrariness of the operators $\mathcal{R}$ and $\mathcal{R}_{1}, \mathcal{R}_{2}$, which in this case may be any homogeneous elliptic differential operator with constant coefficients, and of any order. Moreover, in this case the proof works equally well if it is a pseudo-differential operator. We note that on $\mathbb{R}^{n}$ for some indices explicit expressions for best constants in Sobolev inequalities are available, see e.g. [1,35], and also [17].

Let us give some new examples but, more interestingly, in the setting of the Heisenberg group $\mathbb{H}^{N}$ of homogeneous dimension $Q=2 N+2$. Taking $\mathcal{R}_{1}=\mathcal{R}_{2}=-\Delta_{H}$ the subLaplacian on $\mathbb{H}^{N}, v=2$, for $p=2$ and $a_{1}, a_{2}, m_{1} \in \mathbb{N}, m_{2} \in \mathbb{N} \cup\{0\}$ with $a_{1}=m_{1} \geq$ $a_{2}+1=m_{2}+1$, the Eq. (1.9) becomes a nonlinear differential equation for the poly-subLaplacian

$$
\left(-\Delta_{H}\right)^{m_{1}} u+\left(-\Delta_{H}\right)^{m_{2}} u=|u|^{q-2} u .
$$

Thus, it follows from the results of this paper that for $Q>2 m_{1}$ and $\frac{2 Q}{Q-m_{2} p}<q<\frac{2 Q}{Q-m_{1} p}$, this equation has a ground state $\phi \in L_{m_{1}, m_{2}}^{2}$ minimising the variational problem (1.10), such that $d=\mathfrak{L}(\phi)$ enters the expressions (1.14) and (1.15) for the best constants in the Gagliardo-Nirenberg inequality

$$
\begin{aligned}
& \int_{\mathbb{G}}|u(x)|^{q} d x \\
& \quad \leq C\left(\int_{\mathbb{G}}\left|\left(-\Delta_{H}\right)^{\frac{m_{1}}{2}} u(x)\right|^{2} d x\right)^{\frac{Q(q-2)-2 m_{2} q}{4\left(m_{1}-m_{2}\right)}}\left(\int_{\mathbb{G}}\left|\left(-\Delta_{H}\right)^{\frac{m_{2}}{2}} u(x)\right|^{2} d x\right)^{\frac{2 m_{1} q-Q(q-2)}{4\left(m_{1}-m_{2}\right)}},
\end{aligned}
$$

and in the Sobolev inequality

$$
\|u\|_{L^{q}(\mathbb{G})}^{2} \leq C\left(\left\|\left(-\Delta_{H}\right)^{\frac{m}{2}} u\right\|_{L^{2}(\mathbb{G})}^{2}+\|u\|_{L^{2}(\mathbb{G})}^{2}\right),
$$

with $Q>2 m$ and $2<q<\frac{2 Q}{Q-2 m}$.

Similarly, the $L^{2}$-norms in (1.17) and (1.18) can be replaced by $L^{p}$-norms, see Theorems 3.2 and 5.1, and Theorem 6.1, respectively.

The same is true if we replace the Heisenberg group by any stratified group, or if we replace it by $\mathbb{R}^{n}$ also replacing $\Delta_{H}$ by the Laplacian and $Q$ by $n$.

Some boundary value problems for the poly-sub-Laplacians $\left(-\Delta_{H}\right)^{m}$ have been studied in [25] on the Heisenberg group, and in [26] on general stratified groups. 
The possibility of using different Rockland operators at the same time leads to a wider variety of equations where our results are applicable. For example, let $\left\{X_{j}, Y_{j}\right\}_{j=1}^{N}$ be the basis of the first stratum of a stratified Lie group $\mathbb{G}$. So, this is the case of the Heisenberg group $\mathbb{H}^{N}$, or of $\mathbb{G}=\mathbb{R}^{n}$ with $N=n$. Let us now take

$$
\mathcal{R}_{1}=(-1)^{m_{1}} \sum_{j=1}^{N}\left(X_{j}^{2 m_{1}}+Y_{j}^{2 m_{1}}\right), \quad \mathcal{R}_{2}=(-1)^{m_{2}} \sum_{j=1}^{N} \alpha_{j}\left(X_{j}^{2 m_{2}}+Y_{j}^{2 m_{2}}\right)
$$

for some $\alpha_{j}>0, m_{1}, m_{2} \in \mathbb{N}_{0}, m_{1} \geq m_{2}+1$, so that $v_{1}=2 m_{1}, v_{2}=2 m_{2}$. For $p=2$ the Eq. (1.9) becomes a nonlinear differential equation

$$
(-1)^{m_{1}} \sum_{j=1}^{N}\left(X_{j}^{2 m_{1}}+Y_{j}^{2 m_{1}}\right) u+(-1)^{m_{2}} \sum_{j=1}^{N} \alpha_{j}\left(X_{j}^{2 m_{2}}+Y_{j}^{2 m_{2}}\right) u=|u|^{q-2} u .
$$

Thus, it follows from the results of this paper that for $Q>2 m_{1}$ and $\frac{2 Q}{Q-m_{2} p}<q<\frac{2 Q}{Q-m_{1} p}$, this equation has a ground state $\phi \in L_{m_{1}, m_{2}}^{2}$ minimising the variational problem (1.10), such that $d=\mathfrak{L}(\phi)$ enters the expressions (1.14) and (1.15) for the best constants in the Sobolev inequality (with $m_{2}=0$ )

$$
\|u\|_{L^{q}(\mathbb{G})}^{2} \leq C\left(\left\|\sum_{j=1}^{N}\left(X_{j}^{2 m_{1}}+Y_{j}^{2 m_{1}}\right) u\right\|_{L^{2}(\mathbb{G})}^{2}+\|u\|_{L^{2}(\mathbb{G})}^{2}\right)
$$

and in the Gagliardo-Nirenberg inequality

$$
\begin{aligned}
& \int_{\mathbb{G}}|u(x)|^{q} d x \leq C\left(\int_{\mathbb{G}}\left|\sum_{j=1}^{N}\left(X_{j}^{2 m_{1}}+Y_{j}^{2 m_{1}}\right) u(x)\right|^{2} d x\right)^{\frac{Q(q-2)-2 m_{2} q}{4\left(m_{1}-m_{2}\right)}} \\
& \quad \times\left(\int_{\mathbb{G}}\left|\sum_{j=1}^{N} \alpha_{j}\left(X_{j}^{2 m_{2}}+Y_{j}^{2 m_{2}}\right) u(x)\right|^{2} d x\right)^{\frac{2 m_{1} q-Q(q-2)}{4\left(m_{1}-m_{2}\right)}}
\end{aligned}
$$

respectively.

Weinstein [37] gave the proof of these results in the case $\mathbb{G}=\mathbb{R}^{n}, \mathcal{R}=\mathcal{R}_{1}$ corresponding to the gradient, $p=2$ and $a_{1}=1, a_{2}=0$, by finding the solution to the minimisation problem (1.10). However, his techniques rely on the rearrangement inequalities which are, therefore, specific to $\mathbb{R}^{n}$ in the setting of general nilpotent Lie groups. In the case of $\mathbb{G}$ being the Heisenberg group, $\mathcal{R}=\mathcal{R}_{1}$ corresponding to the horizontal gradient, $p=2$ and $a_{1}=1, a_{2}=0$, these results have been obtained in [5] by a different method relying on obtaining upper and lower estimates on the best constants in the Gagliardo-Nirenberg inequality. This method was effective in several other problems, for example in weighted nonlinear equations [6]. In this paper we will also use this method but now extending it to larger ranges of indices and to general graded Lie groups. On stratified groups, we refer to [33] for the critical case $a_{1}=Q / p$ and $a_{2}=0$ of the Gagliardo-Nirenberg inequality (1.7) and its application to the corresponding Eq. (1.9).

Similar results have been investigated for Riemannian manifolds and hyperbolic spaces, see for example [21] and [16].

The paper is structured as follows. In Sect. 2 we briefly recall further main concepts of graded Lie groups and fix the notation. The Gagliardo-Nirenberg inequality on graded Lie 
group is established in Sect. 3. In Sect. 4 we show the existence of least energy solutions of subelliptic Eq. (1.9). The expressions of the best constant in the Gagliardo-Nirenberg and Sobolev inequalities are obtained in Sects. 5 and 6, respectively.

\section{Preliminaries}

In this section we very briefly recall the necessary notation concerning the setting of graded groups. For a detailed description of the notions of graded and homogeneous nilpotent Lie groups we refer to Folland and Stein [11, Chapter 1], or to the recent exposition in [10, Chapter 3].

A connected simply connected Lie group $\mathbb{G}$ is called a graded Lie group if its Lie algebra $\mathfrak{g}$ admits a gradation

$$
\mathfrak{g}=\bigoplus_{\ell=1}^{\infty} \mathfrak{g}_{\ell}
$$

where the $\mathfrak{g}_{\ell}, \ell=1,2, \ldots$, are vector subspaces of $\mathfrak{g}$, all but finitely many equal to $\{0\}$, and satisfying

$$
\left[\mathfrak{g}_{\ell}, \mathfrak{g}_{\ell^{\prime}}\right] \subset \mathfrak{g}_{\ell+\ell^{\prime}} \quad \forall \ell, \ell^{\prime} \in \mathbb{N} .
$$

Let us now fix a basis $\left\{X_{1}, \ldots, X_{n}\right\}$ of a Lie algebra $\mathfrak{g}$ adapted to the gradation. We obtain points in $\mathbb{G}$ by the exponential mapping $\exp _{\mathbb{G}}: \mathfrak{g} \rightarrow \mathbb{G}$ as

$$
x=\exp _{\mathbb{G}}\left(x_{1} X_{1}+\ldots+x_{n} X_{n}\right) .
$$

A family of dilations of a Lie algebra $\mathfrak{g}$ is a family of linear mappings of the following form

$$
D_{r}=\operatorname{Exp}(A \ln r)=\sum_{k=0}^{\infty} \frac{1}{k !}(\ln (r) A)^{k},
$$

where $A$ is a diagonalisable linear operator on the Lie algebra $\mathfrak{g}$ with positive eigenvalues, and each $D_{r}$ is a morphism of $\mathfrak{g}$, that is, a linear mapping from $\mathfrak{g}$ to itself satisfying:

$$
\forall X, Y \in \mathfrak{g}, r>0,\left[D_{r} X, D_{r} Y\right]=D_{r}[X, Y],
$$

where $[X, Y]:=X Y-Y X$ is the Lie bracket. The dilations can be lifted through the exponential mapping to the group $\mathbb{G}$ by

$$
D_{r}(x)=r x:=\left(r^{\nu_{1}} x_{1}, \ldots, r^{v_{n}} x_{n}\right), \quad x=\left(x_{1}, \ldots, x_{n}\right) \in \mathbb{G}, \quad r>0,
$$

where $v_{1}, \ldots, v_{n}$ are weights of the dilations. The homogeneous dimension of $\mathbb{G}$ is defined by

$$
Q:=\operatorname{Tr} A=v_{1}+\cdots+v_{n} .
$$

We note that the standard Lebesgue measure $d x$ on $\mathbb{R}^{n}$ is the Haar measure for $\mathbb{G}$ (see, e.g. [10, Proposition 1.6.6]). We also recall that a homogeneous quasi-norm on $\mathbb{G}$ is a continuous non-negative function

$$
\mathbb{G} \ni x \mapsto|x| \in[0, \infty)
$$

which satisfies the following properties

- $\left|x^{-1}\right|=|x|$ for all $x \in \mathbb{G}$, 
- $|\lambda x|=\lambda|x|$ for all $x \in \mathbb{G}$ and $\lambda>0$,

- $|x|=0$ if and only if $x=0$.

The quasi-ball centred at $x \in \mathbb{G}$ with radius $R>0$ is defined by

$$
B(x, R):=\left\{y \in \mathbb{G}:\left|x^{-1} y\right|<R\right\} .
$$

Let $\widehat{\mathbb{G}}$ denote the unitary dual of $\mathbb{G}$. For a representation $\pi \in \widehat{\mathbb{G}}$, let $\mathcal{H}_{\pi}^{\infty}$ denote the space of smooth vectors for it. Then a Rockland operator $\mathcal{R}$ on $\mathbb{G}$ is a left-invariant differential operator which is homogeneous of positive degree and satisfies the Rockland condition:

(R) for every representation $\pi \in \widehat{\mathbb{G}}$, except for the trivial representation, the operator $\pi(\mathcal{R})$ is injective on $\mathcal{H}_{\pi}^{\infty}$, that is,

$$
\forall v \in \mathcal{H}_{\pi}^{\infty}, \quad \pi(\mathcal{R}) v=0 \Rightarrow v=0
$$

where $\pi(\mathcal{R}):=d \pi(\mathcal{R})$ is the infinitesimal representation of $\mathcal{R}$ as of an element of the universal enveloping algebra of $\mathbb{G}$.

We refer to [10, Definition 1.7.4 and Section 4.1.1] for a detailed discussion of this definition, that appeared in the work of Rockland [23]. Different characterisations of such operators have been obtained by Rockland [23] and Beals [2], until the resolution in [15] by Helffer and Nourrigat of the so-called Rockland conjecture, which characterised operators satisfying condition $(\mathbf{R})$ as left-invariant homogeneous hypoelliptic differential operators on $\mathbb{G}$.

In this paper we will not be using the representation theoretic interpretation of these operators, so we understand by a Rockland operator any left-invariant homogeneous hypoelliptic differential operator on $\mathbb{G}$. In this paper we will work with the Rockland operators which are positive in the sense of operators.

We refer to [10, Chapter 4] for an extensive presentation concerning Rockland operators and their properties, as well as for the consistent development of the corresponding theory of Sobolev spaces. The corresponding Besov spaces on graded Lie groups and their properties appeared in [7]. On general homogeneous group, we refer to [29] and [31] for Sobolev and Besov type spaces, and to [30] for Morrey and Campanato spaces. A different version of the Gagliardo-Nirenberg inequality on graded Lie groups appeared in [3]. For the pseudo-differential calculus on graded Lie groups, we refer to [10]. Spectral properties of the infinitesimal representations of Rockland operators have been analysed in [36].

\section{Gagliardo-Nirenberg inequality on graded Lie groups}

Let $\mathbb{G}$ be a graded Lie group of homogeneous dimension $Q$ and let $\mathcal{R}_{1}$ and $\mathcal{R}_{2}$ be positive Rockland operators of homogeneous degree $v_{1}$ and $v_{2}$, respectively.

In this section we investigate the Gagliardo-Nirenberg inequality on graded Lie groups. We denote the Sobolev space by $L_{a}^{p}(\mathbb{G})=L_{a, \mathcal{R}}^{p}(\mathbb{G})$, for $a>0$, defined by the norm

$$
\|u\|_{L_{a, \mathcal{R}}^{p}(\mathbb{G})}:=\left(\int_{\mathbb{G}}\left(\left|\mathcal{R}^{\frac{a}{v}} u(x)\right|^{p}+|u(x)|^{p}\right) d x\right)^{1 / p} .
$$

Let us denote

$$
\|u\|_{\dot{L}_{a, \mathcal{R}}^{p}(\mathbb{G})}:=\left\|\mathcal{R}^{a / v} u\right\|_{L^{p}(\mathbb{G})}
$$


We also use the space $L_{a_{1}, a_{2}}^{p}(\mathbb{G})=L_{a_{1}, a_{2}, \mathcal{R}_{1}, \mathcal{R}_{2}}^{p}(\mathbb{G})$, for $a_{1}>a_{2} \geq 0$, defined by the norm

$$
\|u\|_{L_{a_{1}, a_{2}, \mathcal{R}_{1}, \mathcal{R}_{2}}^{p}(\mathbb{G})}:=\left(\int_{\mathbb{G}}\left(\left|\mathcal{R}_{1}^{\frac{a_{1}}{v_{1}}} u(x)\right|^{p}+\left|\mathcal{R}_{2}^{\frac{a_{2}}{\nu_{2}}} u(x)\right|^{p}\right) d x\right)^{1 / p} .
$$

Remark 3.1 We refer to [10, Theorem 4.4.20] for the independence of the spaces $L_{a}^{p}(\mathbb{G})$ of a particular choice of the Rockland operator $\mathcal{R}$. Consequently, the spaces $L_{a_{1}, a_{2}}^{p}(\mathbb{G})=$ $\dot{L}_{a_{1}}^{p}(\mathbb{G}) \cap \dot{L}_{a_{2}}^{p}(\mathbb{G})$ are also independent of the choice of Rockland operators $\mathcal{R}_{1}$ and $\mathcal{R}_{2}$. Note that if $a_{2}=0$, then $L_{a_{1}, 0}^{p}=L_{a_{1}}^{p}(\mathbb{G})$. Let us show this independence and another relation also for $a_{1} \neq a_{2}$. We know that

$$
\left\|\mathcal{R}_{2}^{\frac{a_{2}}{v_{2}}} f\right\|_{L^{p}(\mathbb{G})}<\infty \Longleftrightarrow\left\|\mathcal{R}_{1}^{\frac{a_{2}}{\nu_{1}}} f\right\|_{L^{p}(\mathbb{G})}<\infty
$$

Denoting $\mathcal{R}_{1}^{\frac{a_{2}}{v_{1}}} f=g$, we get

$$
\left\|\mathcal{R}_{1}^{\frac{a_{1}}{\nu_{1}}} f\right\|_{L^{p}(\mathbb{G})}=\left\|\mathcal{R}_{1}^{\frac{a_{1}-a_{2}}{v_{1}}} g\right\|_{L^{p}(\mathbb{G})}<\infty
$$

which gives $g \in \dot{L}_{a_{1}-a_{2}}^{p}(\mathbb{G})$. It follows that $f \in \mathcal{R}_{2}^{-\frac{a_{2}}{v_{1}}}\left(\dot{L}_{a_{1}-a_{2}}^{p}\right)$. Using [10, Theorem 4.4.18] we see again the independence of the spaces $L_{a_{1}, a_{2}}^{p}(\mathbb{G})$ from the choice of Rockland operators $\mathcal{R}_{1}$ and $\mathcal{R}_{2}$.

Thus, we can omit the subscripts $\mathcal{R}$ or $\mathcal{R}_{1}, \mathcal{R}_{2}$ in the notation for these spaces. However, we may sometimes still write these to emphasise the particular norm that we use on these spaces.

In [32], it was shown that if

$$
a>0,1<r<\frac{Q}{a} \text { and } 1 \leq p \leq q \leq \frac{r Q}{Q-a r},
$$

then we have the following Gagliardo-Nirenberg type inequality

$$
\|u\|_{L^{q}(\mathbb{G})} \lesssim\|u\|_{\dot{L}_{a}^{r}(\mathbb{G})}^{s}\|u\|_{L^{p}(\mathbb{G})}^{1-s} \simeq\left\|\mathcal{R}^{a / v} u\right\|_{L^{r}(\mathbb{G})}^{s}\|u\|_{L^{p}(\mathbb{G})}^{1-s},
$$

for $s=\left(\frac{1}{p}-\frac{1}{q}\right)\left(\frac{a}{Q}+\frac{1}{p}-\frac{1}{r}\right)^{-1} \in[0,1]$.

Such inequality was used in [14] and [32] in the analysis of damped wave equations for the sub-Laplacian on the Heisenberg group and Rockland operators on graded Lie groups, respectively. We also refer to [28,29] as well as to the recent book [27] for Sobolev and Caffarelli-Kohn-Nirenberg inequalities on homogeneous groups. For completeness and also to fix the notation and the relation to Sobolev inequalities, we now give a simple proof of this result for the case $p=r$ relevant to our considerations.

Theorem 3.2 Let $\mathbb{G}$ be a graded Lie group of homogeneous dimension $Q$ and let $\mathcal{R}_{1}$ and $\mathcal{R}_{2}$ be positive Rockland operators of homogeneous degrees $v_{1}$ and $v_{2}$, respectively. Let $a_{1}>a_{2} \geq 0,1<p<\frac{Q}{a_{1}}$ and $\frac{p Q}{Q-a_{2} p} \leq q \leq \frac{p Q}{Q-a_{1} p}$. Then there exists a positive constant $C$ such that

$$
\int_{\mathbb{G}}|u(x)|^{q} d x \leq C\left(\int_{\mathbb{G}}\left|\mathcal{R}_{1}^{\frac{a_{1}}{\nu_{1}}} u(x)\right|^{p} d x\right)^{\frac{Q(q-p)-a_{2} p q}{\left(a_{1}-a_{2}\right) p^{2}}}\left(\int_{\mathbb{G}}\left|\mathcal{R}_{2}^{\frac{a_{2}}{\nu_{2}}} u(x)\right|^{p} d x\right)^{\frac{a_{1} p q-Q(q-p)}{\left(a_{1}-a_{2}\right) p^{2}}}
$$

holds for all $u \in \dot{L}_{a_{1}}^{p}(\mathbb{G}) \cap \dot{L}_{a_{2}}^{p}(\mathbb{G})$. 
The proof of Theorem 3.2 will be based on the following Sobolev embedding:

Corollary 3.3 ([10, Proposition 4.4.13, (5)]) Let $\mathbb{G}$ be a graded Lie group of homogeneous dimension $Q$. Let $a>0$ and $1<p<q<\infty$ be such that

$$
Q\left(\frac{1}{p}-\frac{1}{q}\right)=a \text {. }
$$

Then we have

$$
\|u\|_{L^{q}(\mathbb{G})} \lesssim\|u\|_{\dot{L}_{a}^{p}(\mathbb{G})} \simeq\left\|\mathcal{R}^{\frac{a}{v}} u\right\|_{L^{p}(\mathbb{G})},
$$

for all $u \in \dot{L}_{a}^{p}(\mathbb{G})$, where $\mathcal{R}$ is any positive Rockland operator on $\mathbb{G}$ of homogeneous degree v.

We are now ready to prove Theorem 3.2.

Proof of Theorem 3.2 Let us denote $p_{1}=\frac{p Q}{Q-a_{1} p}$ and $p_{2}=\frac{p Q}{Q-a_{2} p}$. Using Hölder's inequality we obtain that

$\int_{\mathbb{G}}|u(x)|^{q} d x=\int_{\mathbb{G}}|u(x)|^{q s}|u(x)|^{q(1-s)} d x \leq\left(\int_{\mathbb{G}}|u(x)|^{p_{1}} d x\right)^{\frac{q s}{p_{1}}}\left(\int_{\mathbb{G}}|u(x)|^{p_{2}} d x\right)^{\frac{q(1-s)}{p_{2}}}$,

where $\frac{q s}{p_{1}}+\frac{q(1-s)}{p_{2}}=1$. Then we find that $s=\frac{Q(q-p)-a_{2} p q}{\left(a_{1}-a_{2}\right) p q}$, so that

$$
\int_{\mathbb{G}}|u(x)|^{q} d x \leq\left(\int_{\mathbb{G}}|u(x)|^{p_{1}} d x\right)^{\frac{Q(q-p)-a_{2} p q}{\left(a_{1}-a_{2}\right) p p_{1}}}\left(\int_{\mathbb{G}}|u(x)|^{p_{2}} d x\right)^{\frac{a_{1} p q-Q(q-p)}{\left(a_{1}-a_{2}\right) p p_{2}}} .
$$

Then, using (3.6) for $\|u\|_{L^{p_{1}(\mathbb{G})}}$ and $\|u\|_{L^{p_{2}(\mathbb{G})}}$ we obtain (3.4).

\section{Existence of least energy solutions for a class of nonlinear subelliptic equations}

In this section we investigate the existence of least energy solutions to a class of nonlinear Schrödinger type equations associated with the positive homogeneous Rockland operators.

So, let operators $\mathcal{R}_{1}$ and $\mathcal{R}_{2}$ be positive Rockland operators of homogeneous degrees $v_{1}$ and $v_{2}$, respectively, on a graded Lie group $\mathbb{G}$ of homogeneous dimension $Q$. Let $a_{1}>a_{2} \geq 0$, $1<p<\frac{Q}{a_{1}}$ and $\frac{p Q}{Q-a_{2} p}<q<\frac{p Q}{Q-a_{1} p}$. Let us consider the following Schrödinger equation with the power nonlinearity

$$
\mathcal{R}_{1}^{\frac{a_{1}}{\nu_{1}}}\left(\left|\mathcal{R}_{1}^{\frac{a_{1}}{\nu_{1}}} u\right|^{p-2} \mathcal{R}_{1}^{\frac{a_{1}}{\nu_{1}}} u\right)+\mathcal{R}_{2}^{\frac{a_{2}}{\nu_{2}}}\left(\left|\mathcal{R}_{2}^{\frac{a_{2}}{\nu_{2}}} u\right|^{p-2} \mathcal{R}_{2}^{\frac{a_{2}}{\nu_{2}}} u\right)=|u|^{q-2} u, \quad u \in L_{a_{1}, a_{2}}^{p}(\mathbb{G}) .
$$

Now, we briefly formulate some notations and definitions.

Definition 4.1 We say that the function $u \in L_{a_{1}, a_{2}}^{p}(\mathbb{G})$ is a solution of (4.1) if and only if for any $\psi \in L_{a_{1}, a_{2}}^{p}(\mathbb{G})$ the identity

$$
\begin{aligned}
& \int_{\mathbb{G}}\left(\left|\mathcal{R}_{1}^{\frac{a_{1}}{\nu_{1}}} u(x)\right|^{p-2} \mathcal{R}_{1}^{\frac{a_{1}}{\nu_{1}}} u(x) \overline{\mathcal{R}_{1}^{\frac{a_{1}}{\nu_{1}}} \psi(x)}+\left|\mathcal{R}_{2}^{\frac{a_{2}}{\nu_{2}}} u(x)\right|^{p-2} \mathcal{R}_{2}^{\frac{a_{2}}{\nu_{2}}} u(x) \overline{\mathcal{R}_{2}^{\frac{a_{2}}{\nu_{2}}} \psi(x)}\right. \\
& \left.\quad-|u(x)|^{q-2} u(x) \overline{\psi(x)}\right) d x=0
\end{aligned}
$$

holds. 
By $\mathfrak{L}: L_{a_{1}, a_{2}}^{p}(\mathbb{G}) \rightarrow \mathbb{R}$ and $\mathfrak{I}: L_{a_{1}, a_{2}}^{p}(\mathbb{G}) \rightarrow \mathbb{R}$ we denote the following functionals acting on $L_{a_{1}, a_{2}}^{p}(\mathbb{G}) \cap L^{q}(\mathbb{G})$ :

$$
\mathfrak{L}(u):=\frac{1}{p} \int_{\mathbb{G}}\left|\mathcal{R}_{1}^{\frac{a_{1}}{v_{1}}} u(x)\right|^{p} d x+\frac{1}{p} \int_{\mathbb{G}}\left|\mathcal{R}_{2}^{\frac{a_{2}}{v_{2}}} u(x)\right|^{p} d x-\frac{1}{q} \int_{\mathbb{G}}|u(x)|^{q} d x
$$

and

$$
\Im(u):=\int_{\mathbb{G}}\left(\left|\mathcal{R}_{1}^{\frac{a_{1}}{\nu_{1}}} u(x)\right|^{p}+\left|\mathcal{R}_{2}^{\frac{a_{2}}{\nu_{2}}} u(x)\right|^{p}-|u(x)|^{q}\right) d x .
$$

We denote the Nehari set by

$$
\mathcal{N}:=\left\{u \in L_{a_{1}, a_{2}}^{p}(\mathbb{G}) \backslash\{0\}: \Im(u)=0\right\}
$$

and we put

$$
d:=\inf \{\mathfrak{L}(u): u \in \mathcal{N}\} .
$$

Definition 4.2 Let $\Gamma$ be the set of the solutions of (4.1), that is,

$$
\Gamma=\left\{\phi \in L_{a_{1}, a_{2}}^{p}(\mathbb{G}): \mathfrak{L}^{\prime}(\phi)=0 \text { and } \phi \neq 0\right\} .
$$

Let $\mathcal{G}$ be the set of least energy solutions of (4.1), namely,

$$
\mathcal{G}=\{u \in \Gamma: \mathfrak{L}(u) \leq \mathfrak{L}(v) \text { for any } v \in \Gamma\} .
$$

Now we state the main result of this section.

Theorem 4.3 Let $a_{1}>a_{2} \geq 0,1<p<\frac{Q}{a_{1}}$ and $\frac{p Q}{Q-a_{2} p}<q<\frac{p Q}{Q-a_{1} p}$. Then the Schrödinger type Eq. (4.1) has a least energy solution $\phi \in L_{a_{1}, a_{2}}^{p}(\mathbb{G})$.

Moreover, we have $d=\mathfrak{L}(\phi)$.

From now on in this section we assume that $a_{1}, a_{2}, p, q$ satisfy conditions of Theorem 4.3. Before proving this theorem let us show the following lemmas.

Lemma 4.4 For all $u \in L_{a_{1}, a_{2}}^{p}(\mathbb{G}) \backslash\{0\}$, there exists a unique $\mu_{u}>0$ such that $\mu_{u} u \in \mathcal{N}$. Moreover, for $\mathfrak{I}(u)<0$ we have $0<\mu_{u}<1$.

Proof of Lemma 4.4 It is easy to see that for an arbitrary $u \in L_{a_{1}, a_{2}}^{p}(\mathbb{G}) \backslash\{0\}$ and for

$$
\mu_{u}=\|u\|_{L_{a_{1}, a_{2}, \mathcal{R}_{1}, \mathcal{R}_{2}}^{p}}^{\frac{p}{q-p}} \quad\|u\|_{L^{q}(\mathbb{G})}^{-\frac{q}{q-p}},
$$

we get $\mu_{u} u \in \mathcal{N}$. Also, it is clear that $\mu_{u}$ is unique. Thus, from the expression for $\mu_{u}$ we obtain that $0<\mu_{u}<1$ provided that $\|u\|_{L_{a_{1}, a_{2}, \mathcal{R}_{1}, \mathcal{R}_{2}}^{p}(\mathbb{G})}^{p}<\|u\|_{L^{q}(\mathbb{G})}^{q}$.

Lemma 4.5 We have $\inf _{u \in \mathcal{N}}\|u\|_{L_{a_{1}, a_{2}, \mathcal{R}_{1}, \mathcal{R}_{2}}^{p}(\mathbb{G})}>0$.

Proof of Lemma 4.5 Using the inequality (3.4), we get for any $u \in \mathcal{N}$, that

$$
\begin{aligned}
\|u\|_{L_{a_{1}, a_{2}, \mathcal{R}_{1}, \mathcal{R}_{2}}^{p}(\mathbb{G})}^{p}=\|u\|_{L^{q}(\mathbb{G})}^{q} & \leq C\left\|\mathcal{R}_{1}^{\frac{a_{1}}{v_{1}}} u\right\|_{L^{p}(\mathbb{G})}^{\frac{Q(q-p)-a_{2} p q}{\left(a_{1}-a_{2}\right) p}}\left\|\mathcal{R}_{2}^{\frac{a_{2}}{v_{2}}} u\right\|_{L^{p}(\mathbb{G})}^{\frac{a_{1} p q-Q(q-p)}{\left(a_{1}-a_{2}\right) p}} \\
& \leq C\|u\|_{L_{a_{1}, a_{2}, \mathcal{R}_{1}, \mathcal{R}_{2}}^{p}(\mathbb{G})}^{\frac{Q(q-p)-a_{2} p q}{\left(a_{1}-a_{2}\right) p}}\|u\|_{L_{a_{1}, a_{2}, \mathcal{R}_{1}, \mathcal{R}_{2}}^{p}}^{\frac{\left.a_{1} p q-Q(q)-p\right)}{\left(a_{1}-a_{2}\right) p}} \\
& =C\|u\|_{L_{a_{1}, a_{2}, \mathcal{R}_{1}, \mathcal{R}_{2}}^{p}(\mathbb{G})}^{q} .
\end{aligned}
$$


Thus, we obtain $\|u\|_{L_{a_{1}, a_{2}, \mathcal{R}_{1}, \mathcal{R}_{2}}^{p-p}(\mathbb{G})}^{q-p} \geq C^{-1}$. Putting $\kappa=C^{-\frac{1}{q-p}}>0$, we observe that $\|u\|_{L_{a_{1}, a_{2}, \mathcal{R}_{1}, \mathcal{R}_{2}}^{p}(\mathbb{G})} \geq \kappa$ for all $u \in \mathcal{N}$.

Let us prove the following Rellich-Kondrachov type lemma. For the Heisenberg group a similar result was proved by Garofalo and Lanconelli [13].

Lemma 4.6 Assume that $a_{1}>a_{2} \geq 0,1<p<\frac{Q}{a_{1}}$ and $\frac{p Q}{Q-a_{2} p}<q<\frac{p Q}{Q-a_{1} p}$. Let $D \subset \mathbb{G}$ be a bounded open set. Then, we get the compact embedding $L_{a_{1}, a_{2}}^{p}(D) \hookrightarrow L^{q}(D)$, where

$$
L_{a_{1}, a_{2}}^{p}(D)=\left\{f \in L_{a_{1}, a_{2}}^{p}(\mathbb{G}): \operatorname{supp} f \subset D\right\} .
$$

Proof of Lemma 4.6 By the density argument, it is enough to prove ${\stackrel{\circ}{a_{1}, a_{2}}}^{p}(D) \hookrightarrow L^{q}(D)$, where $\stackrel{\circ}{L}_{a_{1}, a_{2}}^{p}(D)$ denotes the closure of $C_{0}^{\infty}(D)$ with respect to the norm (3.2).

We set $\phi \in C_{0}^{\infty}(\mathbb{G})$, with $0 \leq \phi \leq 1$, supp $\phi \subset \overline{B(0,1)}$ and $\int_{\mathbb{G}} \phi(x) d x=1$. Let us define

$$
K_{\varepsilon} f:=\phi_{\varepsilon} * f(x),
$$

where $f \in L_{\mathrm{loc}}^{1}(\mathbb{G})$ and $\phi_{\varepsilon}(x):=\varepsilon^{-Q} \phi\left(\varepsilon^{-1} x\right)$ for every $\varepsilon>0$.

We need the following lemma, which is an analogue of [13, Lemma 3.1] for graded groups.

Lemma 4.7 Let $D \subset \mathbb{G}$ be a bounded open set. Let $1<q<\infty$. Then, $Z \subset L^{q}(D)$ is relatively compact in $L^{q}(D)$, if and only if

(1) $Z$ is bounded;

(2) $\left\|K_{\varepsilon} f-f\right\|_{q} \rightarrow 0$ as $\varepsilon \rightarrow 0$, uniformly in $f \in Z$.

Proof of Lemma 4.7 Let us briefly give a sketch of the proof. In order to show the necessity, we extend functions in $L^{q}(D)$ with zero outside $D$. Let us take $f_{1}, \ldots, f_{n}$ from $Z$ and $r>0$, so that the balls in $L^{q}(D)$ centred at $f_{k}$ with radius $r$ cover $Z$. For a given $f$, take $f_{k}$ such that $\left\|f_{k}-f\right\|_{q}<r$. Then we have

$$
\left\|f-K_{\varepsilon} f\right\|_{q} \leq\left\|f-f_{k}\right\|_{q}+\left\|f_{k}-K_{\varepsilon} f_{k}\right\|_{q}+\left\|K_{\varepsilon} f_{k}-K_{\varepsilon} f\right\|_{q} .
$$

Here, taking into account $\left\|K_{\varepsilon} f\right\|_{q} \leq\|f\|_{q}$ and $K_{\varepsilon} f \rightarrow f$ in $L^{q}(D)$ as $\varepsilon \rightarrow 0$ also letting $r \rightarrow 0$, we obtain (2).

Let us now show the sufficiency. Let $f_{n}$ be a bounded sequence in $Z$. Then, by the BanachAlouglu theorem and the reflexivity of $L^{q}, 1<q<\infty$, there exists a subsequence, still denoted by $f_{n}$ weakly convergent in $L^{q}$. In other words, there exists $f \in L^{q}$ such that

$$
\int\left(f_{n}-f\right) h \rightarrow 0, \quad \forall h \in L^{q^{\prime}} .
$$

Now, we show that it actually converges strongly. For this, let us write

$$
\left\|f_{n}-f\right\|_{q} \leq\left\|f_{n}-K_{\varepsilon} f_{n}\right\|_{q}+\left\|K_{\varepsilon} f_{n}-K_{\varepsilon} f\right\|_{q}+\left\|K_{\varepsilon} f-f\right\|_{q} .
$$

Here, the first and third summands vanish when $\varepsilon \rightarrow 0$ due to assumption (2). For the second summand, it follows from (4.8) that for all $x$ and for all $\varepsilon$ we have $K_{\varepsilon}\left(f_{n}-f\right)(x) \rightarrow 0$ as $n \rightarrow \infty$. Since we also have

$$
\left\|\left|K_{\varepsilon}\left(f_{n}-f\right)\right|^{q}\right\|_{L^{1}}=\left\|K_{\varepsilon}\left(f_{n}-f\right)\right\|_{L^{q}}^{q} \leq\left\|\phi_{\varepsilon}\right\|_{L^{1}}^{q}\left\|f_{n}-f\right\|_{L^{q}}^{q}<\infty,
$$


by the Lebesgue dominated convergence theorem we get

$$
\int\left|K_{\varepsilon}\left(f_{n}-f\right)(x)\right|^{q} d x \rightarrow 0
$$

as $n \rightarrow \infty$.

Therefore, from (4.9) we obtain that $Z$ is relatively compact in $L^{q}(D)$.

Let us now come back to the proof of Lemma 4.6. Note that setting $f \equiv 0$ in $\mathbb{G} \backslash D$ for $f \in \stackrel{\circ}{L}_{a_{1}, a_{2}}^{p}(D)$ we get a function in $L_{a_{1}, a_{2}}^{p}(\mathbb{G})$. Now we use Lemma 4.7. Let $Z$ be a bounded set in $\stackrel{p}{L}_{a_{1}, a_{2}}^{p}(D)$. Then, since we have $|B(x, r)|=r^{Q}|B(0,1)|$ for the Haar measure of any open quasi-ball (see e.g. [10, Page 140]), then using the Gagliardo-Nirenberg inequality (3.4) we see that $Z$ is bounded in $L^{q}(D)$.

To complete the proof, we need to verify the second part of Lemma 4.7. For $f \in Z$ by denoting

$$
\psi_{\varepsilon}:=K_{\varepsilon} f-f
$$

and using (3.4), we obtain

$$
\left\|\psi_{\varepsilon}\right\|_{L^{q}(D)} \leq C\left\|\psi_{\varepsilon}\right\|_{\dot{L}_{a_{1}}^{p}(D)}^{\delta}\left\|\psi_{\varepsilon}\right\|_{\dot{L}_{a_{2}}^{p}(D)}^{1-\delta},
$$

where $\delta=\frac{Q(q-p)-a_{2} p q}{\left(a_{1}-a_{2}\right) p}$. Thus, it is enough to show that

$$
\left\|K_{\varepsilon} f-f\right\|_{\dot{L}_{a_{1}}^{p}(D)} \rightarrow 0
$$

that is,

$$
\left\|\mathcal{R}^{\frac{a_{1}}{\nu_{1}}} K_{\varepsilon} f-\mathcal{R}^{\frac{a_{1}}{\nu_{1}}} f\right\|_{L^{p}(D)} \rightarrow 0 .
$$

Indeed, it holds since by [10, Part (i) of Lemma 3.1.58] we have

$$
\mathcal{R}^{\frac{a_{1}}{v_{1}}} K_{\varepsilon} f-\mathcal{R}^{\frac{a_{1}}{v_{1}}} f=\phi_{\varepsilon} * \mathcal{R}^{\frac{a_{1}}{v_{1}}} f-\mathcal{R}^{\frac{a_{1}}{v_{1}}} f \rightarrow 0
$$

as $\varepsilon \rightarrow 0$.

Thus, by Lemma 4.7 we obtain that $Z$ is relatively compact in $L^{q}(D)$.

We also note a property of least energy solutions.

Lemma 4.8 If $v \in \mathcal{N}$ and $\mathfrak{L}(v)=d$ then $v$ must be a least energy solution of the nonlinear Eq. (4.1).

Proof of Lemma 4.8 One can conclude from the Lagrange multiplier rule that there exists a real number $\theta$ such that for an arbitrary $\psi \in L_{a_{1}, a_{2}}^{p}(\mathbb{G})$ we have

$$
\left\langle\mathfrak{L}^{\prime}(v), \psi\right\rangle_{\mathbb{G}}=\theta\left\langle\mathfrak{I}^{\prime}(v), \psi\right\rangle_{\mathbb{G}},
$$

due to the assumption on $v$. Here $\langle\cdot, \cdot\rangle_{\mathbb{G}}$ is a dual product between $L_{a_{1}, a_{2}}^{p}(\mathbb{G})$ and its dual space.

Since $q>p$, we get that

$$
\left\langle\mathfrak{I}^{\prime}(v), v\right\rangle_{\mathbb{G}}=p\|v\|_{L_{a_{1}, a_{2}, \mathcal{R}_{1}, \mathcal{R}_{2}}^{p}(\mathbb{G})}^{p}-q \int_{\mathbb{G}}|v|^{q} d x=(p-q) \int_{\mathbb{G}}|v|^{q} d x<0 .
$$

Also

$$
\left\langle\mathfrak{L}^{\prime}(v), v\right\rangle_{\mathbb{G}}=\mathfrak{I}(v)=0 .
$$


Then due to facts

$$
\left\langle\mathfrak{I}^{\prime}(v), v\right\rangle_{\mathbb{G}}<0
$$

and

$$
\left\langle\mathfrak{L}^{\prime}(v), v\right\rangle_{\mathbb{G}}=0
$$

we obtain that $\theta=0$. Thus, we get $\mathfrak{L}^{\prime}(v)=0$. By taking into account Definition 4.2, we conclude that $v$ is a least energy solution of the nonlinear Eq. (4.1). Lemma 4.8 is proved.

Now we are in a position to prove Theorem 4.3.

Proof of Theorem 4.3 Choose $\left(v_{k}\right)_{k} \subset \mathcal{N}$ as a minimising sequence. By using the Ekeland variational principle we know that there exists a sequence $\left(u_{k}\right)_{k} \subset \mathcal{N}$ such that $\mathfrak{L}\left(u_{k}\right) \rightarrow d$ and $\mathfrak{L}^{\prime}\left(u_{k}\right) \rightarrow 0$. Then, Lemma 4.5 implies that there are two positive constants $C_{1}$ and $C_{2}$ with the properties

$$
C_{1} \leq\left\|u_{k}\right\|_{L_{a_{1}, a_{2}, \mathcal{R}_{1}, \mathcal{R}_{2}}^{p}(\mathbb{G})} \leq C_{2} .
$$

Taking into account this and

$$
\left\|u_{k}\right\|_{L_{a_{1}, a_{2}, \mathcal{R}_{1}, \mathcal{R}_{2}}^{p}}^{p}=\int_{\mathbb{G}}\left|u_{k}(x)\right|^{q} d x,
$$

one gets the existence of a positive constant $C_{3}$ so that we have

$$
\limsup _{k \rightarrow \infty} \int_{\mathbb{G}}\left|u_{k}(x)\right|^{q} d x \geq C_{3}>0 .
$$

By Lemma 4.6 and the concentration compactness argument of [34, Lemma 3.1], we have that $u_{k} \rightarrow 0$ in $L^{q}(\mathbb{G})$ with $\frac{p Q}{Q-a_{2} p}<q<\frac{p Q}{Q-a_{1} p}$ if it is true that

$$
\lim _{k \rightarrow \infty} \sup _{\eta \in \mathbb{G}} \int_{B(\eta, r)}\left|u_{k}(x)\right|^{q} d x=0,
$$

for some $r>0$, where $B(\eta, r)$ is a quasi-ball on $\mathbb{G}$ centred at $\eta$ with radius $r$. Then the fact that there is $C_{4}>0$ and $r>1$ such that

$$
\liminf _{k \rightarrow \infty} \sup _{\eta \in \mathbb{G}} \int_{B(\eta, r)}\left|u_{k}(x)\right|^{q} d x \geq C_{4}>0
$$

follows from the estimate (4.11). We hence may assume that there are $\tilde{x}^{k} \in \mathbb{G}$ with

$$
\liminf _{k \rightarrow \infty} \int_{B\left(\tilde{x}^{k}, r\right)}\left|u_{k}(x)\right|^{q} d x \geq \frac{C_{4}}{2}>0 .
$$

By the bi-invariance of the Haar measure and the left invariance of the operators $\mathcal{R}_{1}, \mathcal{R}_{2}$ we have

$$
\mathfrak{L}\left(u_{k}(\tilde{x} x)\right)=\mathfrak{L}\left(u_{k}(x)\right)
$$

and

$$
\Im\left(u_{k}(\tilde{x} x)\right)=\Im\left(u_{k}(x)\right)
$$


for all $\tilde{x} \in \mathbb{G}$. Let us introduce $\omega_{k}(x):=u_{k}(\tilde{x} x)$. Then we obtain $\mathfrak{L}\left(\omega_{k}\right)=\mathfrak{L}\left(u_{k}\right)$ and $\mathfrak{I}\left(\omega_{k}\right)=\mathfrak{I}\left(u_{k}\right)$. Moreover, it gives the bounded sequence $\left(\omega_{k}\right)_{k}$ of elements of the space $L_{a_{1}, a_{2}}^{p}(\mathbb{G})$ which satisfies

$$
\liminf _{k \rightarrow \infty} \int_{B(0, r)}\left|\omega_{k}(x)\right|^{q} d x \geq \frac{C_{4}}{2}>0 .
$$

There is a subsequence, denoted by $\omega_{k}$ that weakly converges to $\phi$ in the space $L_{a_{1}, a_{2}}^{p}(\mathbb{G})$. Then Lemma 4.6 implies that $\omega_{k}$ strongly converges to $\phi$ in $L_{l o c}^{q}(\mathbb{G})$. Due to this and the estimate (4.15), finally, we obtain that $\phi \neq 0$.

Now we are in a position to show that $\omega_{k}$ converges strongly to $\phi$ in the space $L_{a_{1}, a_{2}}^{p}(\mathbb{G})$. We will show first that $\mathfrak{I}(\phi)=0$. Suppose that $\mathfrak{I}(\phi)<0$. Lemma 4.4 implies that there exists a positive number $\mu_{\phi}<1$ such that $\mu_{\phi} \phi \in \mathcal{N}$ for $\mathfrak{I}(\phi)<0$. Since $\mathfrak{I}\left(\omega_{k}\right)=0$, by the Fatou lemma we get

$$
\begin{aligned}
d+o(1)=\mathfrak{L}\left(\omega_{k}\right) & =\left(\frac{1}{p}-\frac{1}{q}\right) \int_{\mathbb{G}}\left|\omega_{k}(x)\right|^{q} d x \\
& \geq\left(\frac{1}{p}-\frac{1}{q}\right) \int_{\mathbb{G}}|\phi(x)|^{q} d x+o(1) \\
& =\left(\frac{1}{p}-\frac{1}{q}\right) \mu_{\phi}^{-q} \int_{\mathbb{G}}\left|\mu_{\phi} \phi(x)\right|^{q} d x+o(1) \\
& =\mu_{\phi}^{-q} \mathfrak{L}\left(\mu_{\phi} \phi\right)+o(1) .
\end{aligned}
$$

Then, from the property $0<\mu_{\phi}<1$ we obtain that $d>\mathfrak{L}\left(\mu_{\phi} \phi\right)$. Since $\mu_{\phi} \phi \in \mathcal{N}$, we get a contradiction.

Suppose now that $\Im(\phi)>0$. We use the following lemma:

Lemma 4.9 ([4, Lemma 3]) Let $\ell: \mathbb{C} \rightarrow \mathbb{R}$ be convex and let $m>1$. Then

$$
|\ell(a+b)-\ell(a)| \leq \varepsilon[\ell(m a)-m \ell(a)]+\left|\ell\left(C_{\varepsilon} b\right)\right|+\left|\ell\left(-C_{\varepsilon} b\right)\right|
$$

for all $a, b \in \mathbb{C}, 0<\varepsilon<\frac{1}{m}$ and $\frac{1}{C_{\varepsilon}}=\varepsilon(m-1)$.

By Lemma 4.9, as in [4, Example (b), Page 488], we can choose some number $m>1$ such that the hypotheses of [4, Theorem 2] hold for $\varepsilon m<1$ and for $\psi_{k}=\omega_{k}-\phi$ with

$$
\xi_{\varepsilon}(t)=\ell(m t)-m \ell(t) \text { and } \tau_{\varepsilon}(t)=\left|\ell\left(C_{\varepsilon} t\right)\right|+\left|\ell\left(-C_{\varepsilon} t\right)\right|,
$$

where $1 / C_{\varepsilon}=\varepsilon(m-1)$. Therefore, we can use [4, Theorem 2] to obtain

$$
0=\mathfrak{I}\left(\omega_{k}\right)=\mathfrak{I}(\phi)+\Im\left(\psi_{k}\right)+o(1) .
$$

Consequently, we have

$$
\limsup _{k \rightarrow \infty} \mathfrak{I}\left(\psi_{k}\right)<0 .
$$

Using Lemma 4.4, there is a positive constant $\mu_{k}:=\mu_{\psi_{k}}$ with the properties $\mu_{k} \psi_{k} \in \mathcal{N}$ and $\lim \sup \mu_{k} \in(0,1)$. Indeed, suppose that $\lim \sup \mu_{k}=1$. Then we get a subsequence $k \rightarrow \infty$ $\left(\mu_{k_{j}}\right)_{j \in \mathbb{N}}$ such that $\lim _{j \rightarrow \infty} \mu_{k_{j}}=1$. From the property $\mu_{k_{j}} \psi_{k_{j}} \in \mathcal{N}$ we obtain $\Im\left(\psi_{k_{j}}\right)=$ 
$\Im\left(\mu_{k_{j}} \psi_{k_{j}}\right)+o(1)=o(1)$. But this is impossible in view of (4.17). Thus, we have that $\lim \sup \mu_{k} \in(0,1)$. Since $\Im\left(\omega_{k}\right)=0$, as in (4.16) by the Fatou lemma we have $k \rightarrow \infty$

$$
\begin{aligned}
d+o(1) & =\mathfrak{L}\left(\omega_{k}\right)=\left(\frac{1}{p}-\frac{1}{q}\right) \int_{\mathbb{G}}\left|\omega_{k}(x)\right|^{q} d x \\
& \geq\left(\frac{1}{p}-\frac{1}{q}\right) \int_{\mathbb{G}}\left|\psi_{k}(x)\right|^{q} d x+o(1) \\
& =\left(\frac{1}{p}-\frac{1}{q}\right) \mu_{k}^{-q} \int_{\mathbb{G}}\left|\mu_{k} \psi_{k}(x)\right|^{q} d x+o(1) \\
& =\mu_{k}^{-q} \mathfrak{L}\left(\mu_{k} \psi_{k}\right)+o(1) .
\end{aligned}
$$

The fact lim sup $\mu_{k} \in(0,1)$ implies that $d>\mathfrak{L}\left(\mu_{k} \psi_{k}\right)$. It means that we obtain a contradic$k \rightarrow \infty$ tion since $\mu_{k} \psi_{k} \in \mathcal{N}$.

Thus, we must have $\Im(\phi)=0$. Now let us prove that $\psi_{k}=\omega_{k}-\phi \rightarrow 0$ in $L_{a_{1}, a_{2}}^{p}(\mathbb{G})$. Indeed, if it is not true, that is, $\left\|\psi_{k}\right\|_{L_{a_{1}, a_{2}, \mathcal{R}_{1}, \mathcal{R}_{2}}^{p}(\mathbb{G})}$ does not converge to zero as $k \rightarrow \infty$, then there exists a subsequence $\left\{\psi_{k_{n}}\right\}_{n \in \mathbb{N}} \subset\left\{\psi_{k}\right\}_{k \in \mathbb{N}}$, such that $\left\|\psi_{k_{n}}\right\|_{L_{a_{1}, a_{2}, \mathcal{R}_{1}, \mathcal{R}_{2}}(\mathbb{G})} \rightarrow C>0$ as $k \rightarrow \infty$. Using Lemma 4.9 with the same argument as above we get

$$
0=\mathfrak{I}\left(\omega_{k_{n}}\right)=\mathfrak{I}(\phi)+\mathfrak{I}\left(\psi_{k_{n}}\right)+o(1)
$$

which gives $\mathfrak{I}\left(\psi_{k_{n}}\right)=o(1)$. By Lemma 4.4 there exist $\rho_{k_{n}}>0$ such that $\mathfrak{I}\left(\rho_{k_{n}} \psi_{k_{n}}\right)=0$. Moreover, $\rho_{k_{n}} \rightarrow 1$ as $n \rightarrow \infty$. Applying Lemma 4.9 again, we obtain for a large enough $k$ that

$$
\begin{aligned}
d+o(1) & =\mathfrak{L}\left(\omega_{k_{n}}\right)=\mathfrak{L}(\phi)+\mathfrak{L}\left(\psi_{k_{n}}\right)+o(1) \\
& =\mathfrak{L}(\phi)+\mathfrak{L}\left(\rho_{k_{n}} \psi_{k_{n}}\right)+o(1) \\
& \geq d+d+o(1),
\end{aligned}
$$

which is a contradiction because of $d>0$. Thus, we obtain that $\omega_{k}$ converges strongly to $\phi$ in the Sobolev space $L_{a_{1}, a_{2}}^{p}(\mathbb{G})$ and that $\phi$ is a minimiser of $d$. Lemma 4.8 guarantees the fact that $\phi$ is a least energy solution of (4.1).

Theorem 4.3 is proved.

\section{Best constants in the Gagliardo-Nirenberg inequalities}

In this section we obtain a sharp expression for the smallest positive constant $C$ in (3.4). We denote by $C_{G N, \mathcal{R}_{1}, \mathcal{R}_{2}=C_{G N}, \mathcal{R}_{1}, \mathcal{R}_{2}, a_{1}, a_{2}, p, q}$ the smallest positive constant $C$ such that the Gagliardo-Nirenberg inequality (3.4) holds. Now let us show the main result of this section.

Theorem 5.1 Let $a_{1}>a_{2} \geq 0,1<p<\frac{Q}{a_{1}}$ and $\frac{p Q}{Q-a_{2} p}<q<\frac{p Q}{Q-a_{1} p}$. Let $\phi$ be a least energy solution of (4.1) and let $d$ be defined in (4.6). Let $C_{G N, \mathcal{R}_{1}, \mathcal{R}_{2}}$ be the smallest positive constant $C$ in (3.4). Then we have

$$
C_{G N, \mathcal{R}_{1}, \mathcal{R}_{2}}=\frac{\left(a_{1}-a_{2}\right) p q}{a_{1} p q-Q(q-p)}\left(\frac{Q(q-p)-a_{2} p q}{a_{1} p q-Q(q-p)}\right)^{\frac{a_{2} p q-Q(q-p)}{\left(a_{1}-a_{2}\right) p^{2}}}\left(\frac{a_{1} p q-Q(q-p)}{\left(a_{1}-a_{2}\right)(q-p)} d\right)^{\frac{p-q}{p}} .
$$

The proof of Theorem 5.1 will be based on the following lemmas: 
Lemma 5.2 Let $\phi$ be a least energy solution of (4.1). Then we have

$$
\int_{\mathbb{G}}\left|\mathcal{R}_{1}^{\frac{a_{1}}{v_{1}}} \phi(x)\right|^{p} d x=\frac{Q(q-p)-a_{2} p q}{\left(a_{1}-a_{2}\right)(q-p)} d
$$

and

$$
\int_{\mathbb{G}}|\phi(x)|^{q} d x=\frac{p q}{q-p} d .
$$

Proof of Lemma 5.2 Using the fact that $\phi$ be a least energy solution of (4.1), we obtain from (4.2) that

$$
\int_{\mathbb{G}}\left|\mathcal{R}_{1}^{\frac{a_{1}}{\nu_{1}}} \phi(x)\right|^{p} d x+\int_{\mathbb{G}}\left|\mathcal{R}_{2}^{\frac{a_{2}}{\nu_{2}}} \phi(x)\right|^{p} d x=\int_{\mathbb{G}}|\phi(x)|^{q} d x
$$

On the other hand, for $\lambda>0$ and $\widetilde{\phi}_{\lambda}(x):=\lambda^{\frac{Q}{p}} \phi\left(\delta_{\lambda}(x)\right)$ we have the relation

$$
\begin{aligned}
& \mathfrak{L}\left(\widetilde{\phi}_{\lambda}\right)=\frac{\lambda Q}{p} \int_{\mathbb{G}}\left|\mathcal{R}_{1}^{\frac{a_{1}}{\nu_{1}}} \phi\left(\delta_{\lambda}(x)\right)\right|^{p} d x+\frac{\lambda Q}{p} \int_{\mathbb{G}}\left|\mathcal{R}_{2}^{\frac{a_{2}}{\nu_{2}}} \phi\left(\delta_{\lambda}(x)\right)\right|^{p} d x-\frac{\lambda^{\frac{Q q}{p}}}{q} \int_{\mathbb{G}}\left|\phi\left(\delta_{\lambda}(x)\right)\right|^{q} d x \\
& \quad=\frac{\lambda^{a_{1} p}}{p} \int_{\mathbb{G}}\left|\mathcal{R}_{1}^{\frac{a_{1}}{\nu_{1}}} \phi(x)\right|^{p} d x+\frac{\lambda^{a_{2} p}}{p} \int_{\mathbb{G}}\left|\mathcal{R}_{2}^{\frac{a_{2}}{\nu_{2}}} \phi(x)\right|^{p} d x-\frac{\lambda^{\frac{Q q}{p}}-Q}{q} \int_{\mathbb{G}}|\phi(x)|^{q} d x .
\end{aligned}
$$

It follows that

$$
0=\left.\frac{\partial}{\partial \lambda} \mathfrak{L}\left(\widetilde{\phi}_{\lambda}\right)\right|_{\lambda=1}=a_{1} \int_{\mathbb{G}}\left|\mathcal{R}_{1}^{\frac{a_{1}}{\nu_{1}}} \phi(x)\right|^{p} d x+a_{2} \int_{\mathbb{G}}\left|\mathcal{R}_{2}^{\frac{a_{2}}{\nu_{2}}} \phi(x)\right|^{p} d x-\frac{Q(q-p)}{p q} \int_{\mathbb{G}}|\phi(x)|^{q} d x .
$$

Taking into account (3.2) and (4.3), we have by (5.3),

$$
\begin{aligned}
d & =\mathfrak{L}(\phi)=\frac{1}{p}\|\phi\|_{L_{a_{1}, a_{2}, \mathcal{R}_{1}, \mathcal{R}_{2}}^{p}(\mathbb{G})}^{p}-\frac{1}{q}\|\phi\|_{L^{q}(\mathbb{G})}^{q} \\
& =\left(\frac{1}{p}-\frac{1}{q}\right)\|\phi\|_{L^{q}(\mathbb{G})}^{q}=\frac{\left(a_{1}-a_{2}\right)(q-p)}{a_{1} p q-Q(q-p)} \int_{\mathbb{G}}\left|\mathcal{R}_{2}^{\frac{a_{2}}{\nu_{2}}} \phi(x)\right|^{p} d x,
\end{aligned}
$$

so that it follows that

$$
\int_{\mathbb{G}}\left|\mathcal{R}_{2}^{\frac{a_{2}}{v_{2}}} \phi(x)\right|^{p} d x=\frac{a_{1} p q-Q(q-p)}{\left(a_{1}-a_{2}\right)(q-p)} d .
$$

Thus, in combination of the equalities (5.4) and (5.5) we get (5.2) and (5.3).

Lemma 5.3 Let

$$
T_{\rho, p, q}:=\inf \left\{\|u\|_{L_{a_{1}, a_{2}, \mathcal{R}_{1}, \mathcal{R}_{2}}^{p}(\mathbb{G})}^{p}: u \in L_{a_{1}, a_{2}}^{p}(\mathbb{G}) \text { and } \int_{\mathbb{G}}|u(x)|^{q} d x=\rho\right\},
$$

for $\rho>0$. If $\phi$ is a minimiser obtained in Theorem 4.3, then $\phi$ is a minimiser of $T_{\rho_{0}, p, q}$ with $\rho_{0}=\int_{\mathbb{G}}|\phi(x)|^{q} d x$.

Proof of Lemma 5.3 By definition of $T_{\rho_{0}, p, q}$ we can note that $\|\phi\|_{L_{a_{1}, a_{2}, \mathcal{R}_{1}, \mathcal{R}_{2}}^{p}(\mathbb{G})}^{p} \geq T_{\rho_{0}, p, q}$. Now let us show that $T_{\rho_{0}, p, q} \geq\|\phi\|_{L_{a_{1}, a_{2}, \mathcal{R}_{1}, \mathcal{R}_{2}}^{p}(\mathbb{G})^{2}}^{p}$. For all $u \in L_{a_{1}, a_{2}}^{p}(\mathbb{G})$ satisfying $\int_{\mathbb{G}}|u(x)|^{q} d x=\int_{\mathbb{G}}|\phi(x)|^{q} d x$, by Lemma 4.4 there exists a unique

$$
\lambda_{0}=\|u\|_{L_{a_{1}, a_{2}, \mathcal{R}_{1}, \mathcal{R}_{2}}^{p}}^{\frac{p}{q-p}}\left(\int_{\mathbb{G}}|u|^{q} d x\right)^{-\frac{1}{q-p}}
$$


such that $\mathfrak{I}\left(\lambda_{0} u\right)=0$. Using the fact that $\lambda_{0} u \neq 0$ and $\phi$ achieves the minimum $d$, by a direct calculation one has

$$
\begin{aligned}
& \left(\frac{1}{p}-\frac{1}{q}\right)\|\phi\|_{L_{a_{1}, a_{2}, \mathcal{R}_{1}, \mathcal{R}_{2}}^{p}(\mathbb{G})}^{p}=\mathfrak{L}(\phi) \leq \mathfrak{L}\left(\lambda_{0} u\right)=\left(\frac{1}{p}-\frac{1}{q}\right) \lambda_{0}^{p}\|u\|_{L_{a_{1}, a_{2}, \mathcal{R}_{1}, \mathcal{R}_{2}}^{p}}^{p}(\mathbb{G}) \\
& =\left(\frac{1}{p}-\frac{1}{q}\right)\|u\|_{L_{a_{1}, a_{2}, \mathcal{R}_{1}, \mathcal{R}_{2}}^{p-p}(\mathbb{G})}^{\frac{p^{2}}{q-p}}\left(\int_{\mathbb{G}}|u(x)|^{q} d x\right)^{-\frac{p}{q-p}}\|u\|_{L_{a_{1}, a_{2}, \mathcal{R}_{1}, \mathcal{R}_{2}}^{p}}^{p}(\mathbb{G})
\end{aligned}
$$

Then, since $\int_{\mathbb{G}}|u(x)|^{q} d x=\int_{\mathbb{G}}|\phi(x)|^{q} d x$ and $\int_{\mathbb{G}}|\phi(x)|^{q} d x=\|\phi\|_{L_{a_{1}, a_{2}, \mathcal{R}_{1}, \mathcal{R}_{2}}^{p}(\mathbb{G})}^{p}$, we get that $\|\phi\|_{L_{a_{1}, a_{2}, \mathcal{R}_{1}, \mathcal{R}_{2}}^{p}(\mathbb{G})}^{p} \leq\|u\|_{L_{a_{1}, a_{2}, \mathcal{R}_{1}, \mathcal{R}_{2}}^{p}(\mathbb{G})}^{p}$. From the arbitrariness of $u$ we obtain $T_{\rho_{0}, p, q} \geq\|\phi\|_{L_{a_{1}, a_{2}, \mathcal{R}_{1}, \mathcal{R}_{2}}^{p}(\mathbb{G})^{p}}^{p}$. Thus, $T_{\rho_{0}, p, q}=\|\phi\|_{L_{a_{1}, a_{2}, \mathcal{R}_{1}, \mathcal{R}_{2}}^{p}(\mathbb{G})}^{p}$, which gives the fact that $\phi$ is a minimiser of $T_{\rho_{0}, p, q}$.

We are now ready to prove Theorem 5.1.

Proof of Theorem 5.1 Let us denote

$$
\begin{aligned}
J(u): & =\left(\int_{\mathbb{G}}\left|\mathcal{R}_{1}^{\frac{a_{1}}{\nu_{1}}} u(x)\right|^{p} d x\right)^{Q(q-p)-a_{2} p q}\left(\int_{\mathbb{G}}\left|\mathcal{R}_{2}^{\frac{a_{2}}{\nu_{2}}} u(x)\right|^{p} d x\right)^{a_{1} p q-Q(q-p)} \\
& \times\left(\int_{\mathbb{G}}|u(x)|^{q} d x\right)^{-\left(a_{1}-a_{2}\right) p^{2}} .
\end{aligned}
$$

We note that the last functional is defined for all $u \in L_{a_{1}, a_{2}}^{p}(\mathbb{G}) \backslash\{0\}$. Then the sharp expression $C_{G N, \mathcal{R}_{1}, \mathcal{R}_{2}}$ can be estimated as

$$
C_{G N, \mathcal{R}_{1}, \mathcal{R}_{2}}^{-\left(a_{1}-a_{2}\right) p^{2}} \leq J(\phi)
$$

Now let us obtain a lower estimate for the constant $C_{G N, \mathcal{R}_{1}, \mathcal{R}_{2}}^{-\left(a_{1}-a_{2}\right)}$. For positive parameters $\lambda$ and $\mu$, and for any $u \in L_{a_{1}, a_{2}}^{p}(\mathbb{G}) \backslash\{0\}$, we define $\omega(x):=\lambda u\left(\delta_{\mu}(x)\right)$. Then, a direct calculation gives that

$$
\begin{aligned}
& \int_{\mathbb{G}}\left|\mathcal{R}_{1}^{\frac{a_{1}}{\nu_{1}}} \omega(x)\right|^{p} d x=\lambda^{p} \mu^{a_{1} p-Q} \int_{\mathbb{G}}\left|\mathcal{R}_{1}^{\frac{a_{1}}{\nu_{1}}} u(x)\right|^{p} d x, \\
& \int_{\mathbb{G}}\left|\mathcal{R}_{2}^{\frac{a_{2}}{\nu_{2}}} \omega(x)\right|^{p} d x=\lambda^{p} \mu^{a_{2} p-Q} \int_{\mathbb{G}}\left|\mathcal{R}_{2}^{\frac{a_{2}}{\nu_{2}}} u(x)\right|^{p} d x
\end{aligned}
$$

and

$$
\int_{\mathbb{G}}|\omega(x)|^{q} d x=\lambda^{q} \mu^{-Q} \int_{\mathbb{G}}|u(x)|^{q} d x .
$$

We choose $\lambda$ and $\mu$ such that

$$
\lambda^{p} \mu^{a_{2} p-Q} \int_{\mathbb{G}}\left|\mathcal{R}_{2}^{\frac{a_{2}}{\nu_{2}}} u(x)\right|^{p} d x=\int_{\mathbb{G}}\left|\mathcal{R}_{2}^{\frac{a_{2}}{\nu_{2}}} \phi(x)\right|^{p} d x
$$

and

$$
\lambda^{q} \mu^{-Q} \int_{\mathbb{G}}|u(x)|^{q} d x=\int_{\mathbb{G}}|\phi(x)|^{q} d x .
$$


Taking logarithms from (5.12) and (5.13) one can solve the linear equations in $\log \lambda$ and $\log \mu$. One deduces that

$$
\left(Q(q-p)-a_{2} p q\right) \log \lambda=-Q \log \left(\frac{\int_{\mathbb{G}}\left|\mathcal{R}_{2}^{\frac{a_{2}}{v_{2}}} \phi(x)\right|^{p} d x}{\int_{\mathbb{G}}\left|\mathcal{R}_{2}^{\frac{a_{2}}{v_{2}}} u(x)\right|^{p} d x}\right)-\left(a_{2} p-Q\right) \log \left(\frac{\int_{\mathbb{G}}|\phi(x)|^{q} d x}{\int_{\mathbb{G}}|u(x)|^{q} d x}\right)
$$

and

$$
\left(Q(q-p)-a_{2} p q\right) \log \mu=-q \log \left(\frac{\int_{\mathbb{G}}\left|\mathcal{R}_{2}^{\frac{a_{2}}{v_{2}}} \phi(x)\right|^{p} d x}{\int_{\mathbb{G}}\left|\mathcal{R}_{2}^{\frac{a_{2}}{v_{2}}} u(x)\right|^{p} d x}\right)+p \log \left(\frac{\int_{\mathbb{G}}|\phi(x)|^{q} d x}{\int_{\mathbb{G}}|u(x)|^{q} d x}\right) .
$$

By (5.10), (5.11), (5.12), and (5.13), we have the two equalities

$$
\int_{\mathbb{G}}\left|\mathcal{R}_{2}^{\frac{a_{2}}{\nu_{2}}} \omega(x)\right|^{p} d x=\int_{\mathbb{G}}\left|\mathcal{R}_{2}^{\frac{a_{2}}{\nu_{2}}} \phi(x)\right|^{p} d x \text { and } \int_{\mathbb{G}}|\omega(x)|^{q} d x=\int_{\mathbb{G}}|\phi(x)|^{q} d x .
$$

Since $\phi$ is a minimiser of $T_{\rho_{0}, p, q}$ with $\rho_{0}=\int_{\mathbb{G}}|\phi(x)|^{q} d x$ by Lemma 5.3, we obtain that

$$
\int_{\mathbb{G}}\left|\mathcal{R}_{1}^{\frac{a_{1}}{\nu_{1}}} \omega(x)\right|^{p} d x \geq \int_{\mathbb{G}}\left|\mathcal{R}_{1}^{\frac{a_{1}}{\nu_{1}}} \phi(x)\right|^{p} d x
$$

and using (5.9), one gets

$$
\lambda^{p} \mu^{a_{1} p-Q} \int_{\mathbb{G}}\left|\mathcal{R}_{1}^{\frac{a_{1}}{\nu_{1}}} u(x)\right|^{p} d x=\int_{\mathbb{G}}\left|\mathcal{R}_{1}^{\frac{a_{1}}{\nu_{1}}} \omega(x)\right|^{p} d x \geq \int_{\mathbb{G}}\left|\mathcal{R}_{1}^{\frac{a_{1}}{\nu_{1}}} \phi(x)\right|^{p} d x .
$$

Therefore

$$
\begin{array}{r}
\left(Q(q-p)-a_{2} p q\right)\left(p \log \lambda+\left(a_{1} p-Q\right) \log \mu+\log \int_{\mathbb{G}}\left|\mathcal{R}_{1}^{\frac{a_{1}}{\nu_{1}}} u(x)\right|^{p} d x\right) \\
\geq\left(Q(q-p)-a_{2} p q\right) \log \int_{\mathbb{G}}\left|\mathcal{R}_{1}^{\frac{a_{1}}{\nu_{1}}} \phi(x)\right|^{p} d x .
\end{array}
$$

Substituting $\log \lambda$ and $\log \mu$ and a simple rearrangement immediately gives

$$
\log J(u) \geq \log J(\phi) .
$$

Therefore $J$ attains its minimum at $\phi$ and one needs Lemma 5.2 to calculate this minimum.

\section{Best constants in the Sobolev inequalities}

In this section, we investigate the constant $C_{S, \mathcal{R}}=C_{S, \mathcal{R}, a, p, q}$, which is the smallest positive constant in the following Sobolev inequality

$$
\left(\int_{\mathbb{G}}|u(x)|^{q} d x\right)^{\frac{p}{q}} \leq C \int_{\mathbb{G}}\left(\left|\mathcal{R}^{\frac{a}{v}} u(x)\right|^{p}+|u(x)|^{p}\right) d x,
$$

where $u \in L_{a}^{p}(\mathbb{G})$, which is the Sobolev embedding theorem, on graded Lie groups established in [10, Theorem 4.4.28]. Therefore, we can write

$$
C_{S, \mathcal{R}}^{-1}:=\inf _{u \in L_{a}^{p}(\mathbb{G}) \backslash\{0\}} \frac{\int_{\mathbb{G}}\left(\left|\mathcal{R}^{\frac{a}{v}} u(x)\right|^{p}+|u(x)|^{p}\right) d x}{\left(\int_{\mathbb{G}}|u(x)|^{q} d x\right)^{\frac{p}{q}}} .
$$


Theorem 6.1 Let $a>0,1<p<\frac{Q}{a}$ and $p<q<\frac{p Q}{Q-a p}$. Let $\phi$ be a least energy solution of (4.1) (with $a_{1}=a, \mathcal{R}_{1}=\mathcal{R}$, and $a_{2}=0$ ) and let $\stackrel{Q}{C}, \mathcal{R}$ be the smallest positive constant $C$ in (6.1). Then we have

$$
C_{S, \mathcal{R}}=\left(\frac{a p q}{a p q-Q(q-p)} \int_{\mathbb{G}}|\phi(x)|^{p} d x\right)^{\frac{p-q}{q}}=\left(\frac{p q}{q-p} d\right)^{\frac{p-q}{q}},
$$

where $d$ is defined in (4.6).

Proof of Theorem 1.6 Using Lemma 5.2 for $a_{1}=a, a_{2}=0, \mathcal{R}_{1}=\mathcal{R}, v_{1}=v$, and $\Im(\phi)=0$, one calculates

$$
\frac{\int_{\mathbb{G}}\left(\left|\mathcal{R}^{\frac{a}{v}} \phi(x)\right|^{p}+|\phi(x)|^{p}\right) d x}{\left(\int_{\mathbb{G}}|\phi(x)|^{q} d x\right)^{\frac{p}{q}}}=\left(\int_{\mathbb{G}}|\phi(x)|^{q}\right)^{\frac{q-p}{q}}=\left(\frac{a p q}{a p q-Q(q-p)} \int_{\mathbb{G}}|\phi(x)|^{p} d x\right)^{\frac{q-p}{q}} .
$$

It follows that

$$
C_{S, \mathcal{R}}^{-1} \leq\left(\frac{a p q}{a p q-Q(q-p)} \int_{\mathbb{G}}|\phi(x)|^{p} d x\right)^{\frac{q-p}{q}} .
$$

For all $u \in L_{a}^{p}(\mathbb{G}) \backslash\{0\}$, we denote $\tilde{u}(x):=\|\phi\|_{L^{q}(\mathbb{G})}\|u\|_{L^{q}(\mathbb{G})}^{-1} u(x)$ so that

$$
\int_{\mathbb{G}}|\tilde{u}(x)|^{q} d x=\int_{\mathbb{G}}|\phi(x)|^{q} d x .
$$

Then, by Lemma 5.3 for $a_{1}=a, a_{2}=0$ and $\mathcal{R}_{1}=\mathcal{R}$ we obtain that

$$
\begin{aligned}
& \int_{\mathbb{G}}\left(\left|\mathcal{R}^{\frac{a}{v}} \tilde{u}(x)\right|^{p}+|\widetilde{u}(x)|^{p}\right) d x \geq \int_{\mathbb{G}}\left(\left|\mathcal{R}^{\frac{a}{v}} \phi(x)\right|^{p}+|\phi(x)|^{p}\right) d x \\
& \quad=\frac{a p q}{a p q-Q(q-p)} \int_{\mathbb{G}}|\phi(x)|^{p} d x,
\end{aligned}
$$

the last equality holds in view of (5.2) for $a_{1}=a, a_{2}=0$ and $\mathcal{R}_{1}=\mathcal{R}$. In the case $a_{1}=a$ and $a_{2}=0$, by (5.3) we obtain

$$
\begin{aligned}
& \frac{\int_{\mathbb{G}}\left(\left|\mathcal{R}^{\frac{a}{v}} u(x)\right|^{p}+|u(x)|^{p}\right) d x}{\left(\int_{\mathbb{G}}|u(x)|^{q} d x\right)^{\frac{p}{q}}} \geq \frac{a p q}{a p q-Q(q-p)} \int_{\mathbb{G}}|\phi(x)|^{p} d x\left(\int_{\mathbb{G}}|\phi(x)|^{q} d x\right)^{-\frac{p}{q}} \\
& \quad=\left(\frac{a p q}{a p q-Q(q-p)} \int_{\mathbb{G}}|\phi(x)|^{p} d x\right)^{1-\frac{p}{q}} .
\end{aligned}
$$

Thus, the estimates (6.4) and (6.5) imply the first equality in (6.3). Putting (5.6) in the first equality of (6.3) for $a_{1}=a$ and $a_{2}=0$, we obtain the second equality in (6.3).

Remark 6.2 From Theorems 5.1 and 6.1, we note that the best constants in the Sobolev and Gagliardo-Nirenberg (with $a_{2}=0$ ) inequalities are related by

$$
C_{G N, \mathcal{R}, a, 0}^{\frac{p}{q}}=C_{S, \mathcal{R}} \frac{a p q}{a p q-Q(q-p)}\left(\frac{Q(q-p)}{a p q-Q(q-p)}\right)^{\frac{Q(p-q)}{a p q}} .
$$

It is interesting to note that while each best constant depends on the positive Rockland operator $\mathcal{R}$ used in the definition of the norm, the ratio $C_{G N, \mathcal{R}, a, 0}^{\frac{p}{q}} / C_{S, \mathcal{R}}$ is independent of $\mathcal{R}$.

Acknowledgements The authors would like to thank the anonymous referees for their helpful and constructive comments that greatly contributed to improving the final version of the paper. 
Open Access This article is licensed under a Creative Commons Attribution 4.0 International License, which permits use, sharing, adaptation, distribution and reproduction in any medium or format, as long as you give appropriate credit to the original author(s) and the source, provide a link to the Creative Commons licence, and indicate if changes were made. The images or other third party material in this article are included in the article's Creative Commons licence, unless indicated otherwise in a credit line to the material. If material is not included in the article's Creative Commons licence and your intended use is not permitted by statutory regulation or exceeds the permitted use, you will need to obtain permission directly from the copyright holder. To view a copy of this licence, visit http://creativecommons.org/licenses/by/4.0/.

\section{References}

1. Aubin, T.: Problèmes isopérimétriques et espaces de Sobolev. J. Differ. Geom. 11(4), 573-598 (1976)

2. Beals, R.: Opérateurs invariants hypoelliptiques sur un groupe de Lie nilpotent. Séminaire GoulaouicSchwartz 1976/1977: Équations aux dérivées partielles et analyse fonctionnelle, Exp. No. 19, 8 pp (1977)

3. Bahouri, H., Fermanian-Kammerer, C., Gallagher, I.: Refined inequalities on graded Lie groups. C. R. Math. Acad. Sci. Paris 350(7-8), 393-397 (2012)

4. Brézis, H., Lieb, E.H.: A relation between pointwise convergence of functions and convergence of functionals. Proc. Am. Math. Soc. 88(3), 486-490 (1983)

5. Chen, J., Rocha, E.M.: A class of sub-elliptic equations on the Heisenberg group and related interpolation inequalities. In: Advances in Harmonic Analysis and Operator Theory, Volume 229 of Operator Theory: Advances and Applications, pp. 123-137. Birkhäuser/Springer Basel AG, Basel (2013)

6. Chen, J., Rocha, E.M.: Existence of solution of sub-elliptic equations on the Heisenberg group with critical growth and double singularities. Opuscula Math. 33(2), 237-254 (2013)

7. Cardona, D., Ruzhansky, M.: Multipliers for Besov spaces on graded Lie groups. C. R. Math. Acad. Sci. Paris 355(4), 400-405 (2017)

8. Folland, G.B.: Subelliptic estimates and function spaces on nilpotent Lie groups. Ark. Mat. 13(2), 161-207 (1975)

9. Fischer, V., Ruzhansky, M.: Sobolev spaces on graded groups. Ann. Inst. Fourier (Grenoble) 67(4), 16711723 (2017)

10. Fischer, V., Ruzhansky, M.: Quantization on Nilpotent Lie Groups, Volume 314 of Progress in Mathematics. Birkhäuser/Springer [Open access book] (2016)

11. Folland, G.B., Stein, E.M.: Hardy Spaces on Homogeneous Groups, Volume 28 of Mathematical Notes. Princeton University Press, Princeton, N.J.; University of Tokyo Press, Tokyo (1982)

12. Gagliardo, E.: Ulteriori proprietà di alcune classi di funzioni in più variabili. Ricerche Mat. 8, 24-51 (1959)

13. Garofalo, N., Lanconelli, E.: Existence and nonexistence results for semilinear equations on the Heisenberg group. Indiana Univ. Math. J. 41(1), 71-98 (1992)

14. Georgiev, V., Palmieri, A.: Critical exponent of Fujita-type for the semilinear damped wave equation on the Heisenberg group with power nonlinearity. J. Differ. Equ. 269(1), 420-448 (2020)

15. Helffer, B., Nourrigat, J.: Caracterisation des opérateurs hypoelliptiques homogènes invariants à gauche sur un groupe de Lie nilpotent gradué. Commun. Partial Differ. Equ. 4(8), 899-958 (1979)

16. Hebey, E., Vaugon, M.: The best constant problem in the Sobolev embedding theorem for complete Riemannian manifolds. Duke Math. J. 79(1), 235-279 (1995)

17. Lieb, E.H., Loss, M.: Analysis, Volume 14 of Graduate Studies in Mathematics, 2nd edn. American Mathematical Society, Providence, RI (2001)

18. Miller, K.G.: Parametrices for hypoelliptic operators on step two nilpotent Lie groups. Commun. Partial Differ. Equ. 5(11), 1153-1184 (1980)

19. Merle, F., Raphael, P.: On universality of blow-up profile for $L^{2}$ critical nonlinear Schrödinger equation. Invent. Math. 156(3), 565-672 (2004)

20. Merle, F., Raphael, P.: Profiles and quantization of the blow up mass for critical nonlinear Schrödinger equation. Commun. Math. Phys. 253(3), 675-704 (2005)

21. Mancini, G., Sandeep, K.: Extremals for Sobolev and exponential inequalities in hyperbolic space. Concentration Analysis and Applications to PDE. volume 229 of Trends in Mathematics, pp. 49-67. Birkhäuser/Springer Basel AG, Basel (2013)

22. Nirenberg, L.: On elliptic partial differential equations. Ann. Scuola Norm. Sup. Pisa 3(13), 115-162 (1959)

23. Rockland, C.: Hypoellipticity on the Heisenberg group-representation-theoretic criteria. Trans. Am. Math. Soc. 240, 1-52 (1978) 
24. Rothschild, L.P., Stein, E.M.: Hypoelliptic differential operators and nilpotent groups. Acta Math. 137(34), 247-320 (1976)

25. Ruzhansky, M., Suragan, D.: On Kac's principle of not feeling the boundary for the Kohn Laplacian on the Heisenberg group. Proc. Am. Math. Soc. 144(2), 709-721 (2016)

26. Ruzhansky, M., Suragan, D.: Layer potentials, Kac's problem, and refined Hardy inequality on homogeneous Carnot groups. Adv. Math. 308, 483-528 (2017)

27. Ruzhansky, M., Suragan, D.: Hardy Inequalities on Homogeneous Groups, Volume 327 of Progress in Mathematics. Birkhäuser/Springer [Open access book] (2019)

28. Ruzhansky, M., Suragan, D., Yessirkegenov, N.: Extended Caffarelli-Kohn-Nirenberg inequalities and superweights for $L^{p}$-weighted Hardy inequalities. C. R. Math. Acad. Sci. Paris 355(6), 694-698 (2017)

29. Ruzhansky, M., Suragan, D., Yessirkegenov, N.: Sobolev type inequalities, Euler-Hilbert-Sobolev and Sobolev-Lorentz-Zygmund spaces on homogeneous groups. Integral Equ. Oper. Theory (2018). https:// doi.org/10.1007/s00020-018-2437-7

30. Ruzhansky, M., Suragan, D., Yessirkegenov, N.: Hardy-Littlewood, Bessel-Riesz, and fractional integral operators in anisotropic Morrey and Campanato spaces. Fract. Calc. Appl. Anal. 21(3), 577-612 (2018)

31. Ruzhansky, M., Suragan, D., Yessirkegenov, N.: Euler semigroup, Hardy-Sobolev and GagliardoNirenberg type inequalities on homogeneous groups. Semigroup Forum 101, 162-191 (2020)

32. Ruzhansky, M., Tokmagambetov, N.: Nonlinear damped wave equations for the sub-Laplacian on the Heisenberg group and for Rockland operators on graded Lie groups. J. Differ. Equ. 265(10), 5212-5236 (2018)

33. Ruzhansky, M., Yessirkegenov, N.: Limiting cases of Sobolev inequalities on stratified groups. Proc. Jpn. Acad. Ser. A 95(8), 83-87 (2019)

34. Schindler, I., Tintarev, K.: An abstract version of the concentration compactness principle. Rev. Mat. Complut. 15(2), 417-436 (2002)

35. Talenti, G.: Best constant in Sobolev inequality. Ann. Mat. Pura Appl. 4(110), 353-372 (1976)

36. ter Elst, A.F.M., Robinson, D.W.: Spectral estimates for positive Rockland operators. In: Algebraic Groups and Lie Groups, Volume 9 of Austral. Mathematical Society Lecture Series, pp. 195-213. Cambridge University Press, Cambridge (1997)

37. Weinstein, M.I.: Nonlinear Schrödinger equations and sharp interpolation estimates. Commun. Math. Phys. 87(4), 567-576 (1982/1983)

Publisher's Note Springer Nature remains neutral with regard to jurisdictional claims in published maps and institutional affiliations.

\section{Affiliations}

\section{Michael Ruzhansky ${ }^{1,2} \cdot$ Niyaz Tokmagambetov $^{1,3} \cdot$ Nurgissa Yessirkegenov $^{1,4,5}$}

Niyaz Tokmagambetov

Niyaz.Tokmagambetov@UGent.be

Nurgissa Yessirkegenov

nurgissa.yessirkegenov@gmail.com

1 Department of Mathematics: Analysis, Logic and Discrete Mathematics, Ghent University, 281

Krijgslaan, Building S8, Ghent, Belgium

2 School of Mathematical Sciences, Queen Mary University of London, London, UK

3 Al-Farabi Kazakh National University, 71 al-Farabi Ave., Almaty 050040, Kazakhstan

4 Institute of Mathematics and Mathematical Modelling, 125 Pushkin Str., Almaty 050010, Kazakhstan

5 Department of Mathematics, Imperial College London, 180 Queen's Gate, London SW7 2AZ, UK 